\title{
OBLIQUITIES OF HOT JUPITER HOST STARS: EVIDENCE FOR TIDAL INTERACTIONS AND PRIMORDIAL MISALIGNMENTS*
}

\author{
Simon Albrecht ${ }^{1}$, Joshua N. Winn ${ }^{1}$, John A. Johnson ${ }^{2,3}$, Andrew W. Howard ${ }^{4}$, Geoffrey W. Marcy ${ }^{4}$, \\ R. Paul Butler ${ }^{5}$, Pamela Arriagada ${ }^{6}$, Jeffrey D. Crane ${ }^{7}$, Stephen A. Shectman $^{7}$, Ian B. Thompson ${ }^{7}$, \\ Teruyuki Hirano $^{8}$, Gaspar Bakos ${ }^{9,10}$, and Joel D. Hartman ${ }^{9}$ \\ ${ }^{1}$ Department of Physics, and Kavli Institute for Astrophysics and Space Research, Massachusetts Institute of Technology, Cambridge, MA 02139, USA \\ ${ }^{2}$ Department of Astrophysics, California Institute of Technology, MC249-17, Pasadena, CA 91125, USA \\ ${ }^{3}$ NASA Exoplanet Science Institute (NExScI), CIT Mail Code 100-22, 770 South Wilson Avenue, Pasadena, CA 91125, USA \\ ${ }^{4}$ Department of Astronomy, University of California, Berkeley, CA 94720, USA \\ ${ }^{5}$ Department of Terrestrial Magnetism, Carnegie Institution of Washington, 5241 Broad Branch Road NW, Washington, DC 20015, USA \\ ${ }^{6}$ Department of Astronomy, Pontificia Universidad Católica de Chile, Casilla 306, Santiago 22, Chile \\ ${ }^{7}$ The Observatories of the Carnegie Institution of Washington, 813 Santa Barbara Street, Pasadena, CA 91101, USA \\ ${ }^{8}$ Department of Physics, The University of Tokyo, Tokyo 113-0033, Japan \\ ${ }^{9}$ Department of Astrophysical Sciences, Princeton University, Peyton Hall, Princeton, NJ 08544, USA \\ Received 2012 April 19; accepted 2012 July 18; published 2012 August 30
}

\begin{abstract}
We provide evidence that the obliquities of stars with close-in giant planets were initially nearly random, and that the low obliquities that are often observed are a consequence of star-planet tidal interactions. The evidence is based on 14 new measurements of the Rossiter-McLaughlin effect (for the systems HAT-P-6, HAT-P-7, HAT-P-16, HAT-P-24, HAT-P-32, HAT-P-34, WASP-12, WASP-16, WASP-18, WASP-19, WASP-26, WASP-31, Gl 436, and Kepler-8), as well as a critical review of previous observations. The low-obliquity (well-aligned) systems are those for which the expected tidal timescale is short, and likewise the high-obliquity (misaligned and retrograde) systems are those for which the expected timescale is long. At face value, this finding indicates that the origin of hot Jupiters involves dynamical interactions like planet-planet interactions or the Kozai effect that tilt their orbits rather than inspiraling due to interaction with a protoplanetary disk. We discuss the status of this hypothesis and the observations that are needed for a more definitive conclusion.
\end{abstract}

Key words: planetary systems - planets and satellites: formation - planet-star interactions - stars: rotation techniques: spectroscopic

Online-only material: color figures, machine-readable table

\section{INTRODUCTION}

Exoplanetary science has been full of surprises. One of the biggest surprises that emerged at the dawn of this field is the existence of "hot Jupiters" having orbital distances much smaller than an astronomical unit (AU). It is thought that giant planets can only form at distances of several AU from their host stars, where the environment is cooler and solid particles are more abundant, facilitating the growth of rocky cores that can then attract gaseous envelopes from the protoplanetary disk.

Different mechanisms have been proposed which might transport giant planets from their presumed birthplaces inward to where we find them. Among the differences between the proposed mechanisms is that some of them would alter the planet's orbital orientation and thereby change the relative orientation between the stellar and orbital spins (Nagasawa et al. 2008; Fabrycky \& Tremaine 2007; Naoz et al. 2011), while others would preserve the relative orientation (Lin et al. 1996), or even reduce any primordial misalignment (Cresswell et al. 2007). For this reason, measuring the stellar obliquity-the angle between stellar and orbital axes-has attracted attention as a possible means of distinguishing between different theories for the origin of hot Jupiters.

\footnotetext{
* The data presented herein were collected with the Magellan (Clay) Telescope located at Las Campanas Observatory, Chile, and the Keck I telescope at the W. M. Keck Observatory, which is operated as a scientific partnership among the California Institute of Technology, the University of California, and the National Aeronautics and Space Administration.

${ }^{10}$ Alfred. P. Sloan Fellow.
}

The stellar obliquity is an elusive parameter because the stellar surface needs to be at least partially resolved by the observer. If the system exhibits eclipses or transits, then it is possible to detect anomalies in the spectral absorption lines of the eclipsed star, which have their origin in the partial blockage of the rotating photosphere. The precise manifestation of the rotation anomaly depends on the angle between the projections of the stellar rotation axis and orbital axis of the occulting companion. Credit for the first definitive measurements has been apportioned to Rossiter (1924) and McLaughlin (1924), after whom the effect is now named. Queloz et al. (2000) were the first to measure the Rossiter-McLaughlin (RM) effect for a planet-hosting star, finding a low obliquity. Since then many systems have been studied, including those with misaligned stars (Hébrard et al. 2008), retrograde orbits (Winn et al. 2009; Triaud et al. 2010), a hot Neptune (Winn et al. 2010c; Hirano et al. 2011b), and even a circumbinary planet (Winn et al. 2011a).

Winn et al. (2010a) found a possible pattern in the hot Jupiter data, namely, host stars hotter than $T_{\text {eff }} \approx 6250 \mathrm{~K}$ tend to have high obliquities, while cooler stars have low obliquities. Schlaufman (2010), using a different method, also found a preponderance of high obliquities among hot stars. Winn et al. (2010a) speculated that this pattern was due to tidal interactions. Specifically, it was hypothesized that hot Jupiters are transported inward by processes that perturb orbital inclinations and lead to a very broad range of obliquities. Cool stars ultimately come into alignment with the orbits because they have higher rates of tidal dissipation due to their thick convective envelopes. Hot 
Table 1

Observation Log

\begin{tabular}{llc}
\hline \hline System & \multicolumn{1}{c}{ Observation Night } & Spectrograph \\
\hline HAT-P-6 & 2010 Nov 5/6 & \\
HAT-P-7 & 2010 Jul 23/24 & HIRES \\
HAT-P-16 & 2010 Dec 26/27 & HIRES \\
HAT-P-24 & 2010 Sep 27/28 & HIRES \\
HAT-P-32 & 2011 Dec 5/6 & HIRES \\
HAT-P-34 & 2011 Sep 2/3 & HIRES \\
WASP-12 & 2012 Jan $1 / 2$ & PFS \\
WASP-16 & 2010 Jul 3/4 & PFS \\
WASP-18 & 2011 Oct 8/9 & PFS \\
WASP-19 & 2010 May 20/21 & PFS \\
WASP-26 & 2010 Aug $17 / 18$ & HIRES \\
WASP-31 & 2012 Mar $12 / 13$ & HIRES \\
Gl 436 & 2010 Apr 24/25 & HIRES \\
Kepler-8 & 2011 Aug 7/8 &
\end{tabular}

stars, in contrast, lack thick convective envelopes and are unable to reorient completely on Gyr timescales.

If this interpretation is correct, then not only should further measurements be consistent with the hot/cool pattern, but also the degree of alignment should be found to correlate with the orbital period and planet-to-star mass ratio, parameters which also strongly affect the rate of tidal dissipation. Since the study by Winn et al. (2010a) the number of systems with RM measurements has nearly doubled. Here we present the results of an additional 14 observations, as well as a critical review of other published measurements (including thorough re-analyses in three cases). We can now attempt a comparison between the measured obliquities and the theoretical distribution of obliquities that one would expect if tides were an important factor. We also refer the reader to Hansen (2012), who recently performed a comparison with a similar motivation, without the benefit of the new RM measurements presented here, but using more sophisticated theoretical models and drawing qualitatively similar conclusions.

This paper has two main parts. The first part is observational. We describe our new observations of the RM effect (Section 2), our analysis method (Section 3), and the details of individual systems analyzed here (Section 4). The second part (Section 5) considers the distribution of obliquities, seeks evidence for the expected signatures of tidal effects, and considers the implications for the origin of hot Jupiters. We summarize our results in Section 6.

\section{OBSERVATIONS}

The 14 new observations presented in this paper were conducted with the Keck I telescope and its High Resolution Spectrograph (HIRES; Vogt et al. 1994), for northern objects, and with the Magellan II (Clay) $6.5 \mathrm{~m}$ telescope and the Planet Finder Spectrograph (PFS; Crane et al. 2010) for southern objects. Table 1 is a $\log$ of the observations. To derive the relative radial velocities (RVs) we compared the spectra observed through the iodine cell with the stellar template spectrum multiplied by an iodine template spectrum. The velocity shift of the stellar template and the parameters of the point-spread function (PSF) of the spectrograph are free parameters in this comparison. The velocity shift of the template that gives the best fit to an observed spectrum represents the measured relative RV for that observation. In particular, we used codes based on those of Butler et al.
Table 2

Relative Radial Velocity Measurements

\begin{tabular}{lcccc}
\hline \hline System & $\begin{array}{c}\text { Time } \\
{\left[\mathrm{BJD}_{\text {TDB }}\right]}\end{array}$ & $\begin{array}{c}\text { RV } \\
\left(\mathrm{m} \mathrm{s}^{-1}\right)\end{array}$ & $\begin{array}{c}\text { Unc. } \\
\left(\mathrm{m} \mathrm{s}^{-1}\right)\end{array}$ & Spectrograph \\
\hline HAT-P-6 & 2455526.70653 & 16.00 & 4.97 & HIRES \\
HAT-P-6 & 2455526.71094 & 35.72 & 5.25 & HIRES \\
HAT-P-6 & 2455526.71507 & 22.91 & 5.13 & HIRES \\
HAT-P-6 & 2455526.71907 & -1.16 & 5.01 & HIRES \\
HAT-P-6 & 2455526.72347 & -2.27 & 4.68 & HIRES \\
HAT-P-6 & 2455526.72775 & -14.90 & 4.63 & HIRES \\
HAT-P-6 & 2455526.73174 & -14.49 & 4.50 & HIRES \\
& & $:$ & & \\
& & $:$ & & \\
\hline
\end{tabular}

(This table is available in its entirety in a machine-readable form in the online journal. A portion is shown here for guidance regarding its form and content.)

(1996). The RVs used in this paper for all system are presented in Table 2.

\section{ANALYSIS OF THE ROSSITER-McLAUGHLIN EFFECT}

In the following sections we describe our strategy to obtain projected obliquities from the observed RVs. Our goal was to perform a relatively simple and homogeneous analysis and thereby facilitate comparisons between systems in the sample. For some systems for which we obtained new data, RM measurements have already been published by other authors. In order to obtain independent measurements and compare the results, in most cases we did not include the previous data in our analysis.

Observing the RM effect allows one to measure the projected angle between the orbital and stellar spins $(\lambda)$ and the projected stellar rotation speed $\left(v \sin i_{\star}\right)$. Here $v$ indicates the stellar rotation speed and $i_{\star}$ the inclination of the stellar spin axis toward the observer. Observing the RM effect provides information on the transit geometry because the distortion of the stellar absorption line-specifically its net redshift or blueshift-depends on the RV of the hidden portion of the stellar photosphere.

For example, if the obliquity were low, then the planet would begin the transit on the approaching half of the photosphere. The blockage causes the absorption lines to appear slightly redshifted. During the second half of the transit, the reverse would be true: the anomalous RV would be a blueshift. In contrast, if the planet's trajectory were entirely over the redshifted half of the star, then the RM effect would be a blueshift throughout the transit. For a retrograde configuration, the RM effect would first lead to a blueshift and then a redshift.

In Sections 3.1-3.5, we discuss some specific effects which influence the RM signal and how we included these in our modeling. Section 3.6 gives the details of our quantitative model of the RM effect, and the prior information about each system that is used in the analysis.

\subsection{Line Broadening}

In addition to stellar rotation, microturbulence and macroturbulence will affect stellar absorption lines and should be considered in the description of the RM effect. Microturbulence is well described by a convolution of the rotationally broadened line with a Gaussian function. Describing macroturbulence is more complex, as it depends on the angle between the line of sight and the local surface normal. Near the center of the stellar disk, the Doppler shifts are produced by vertical ("radial") motions, 

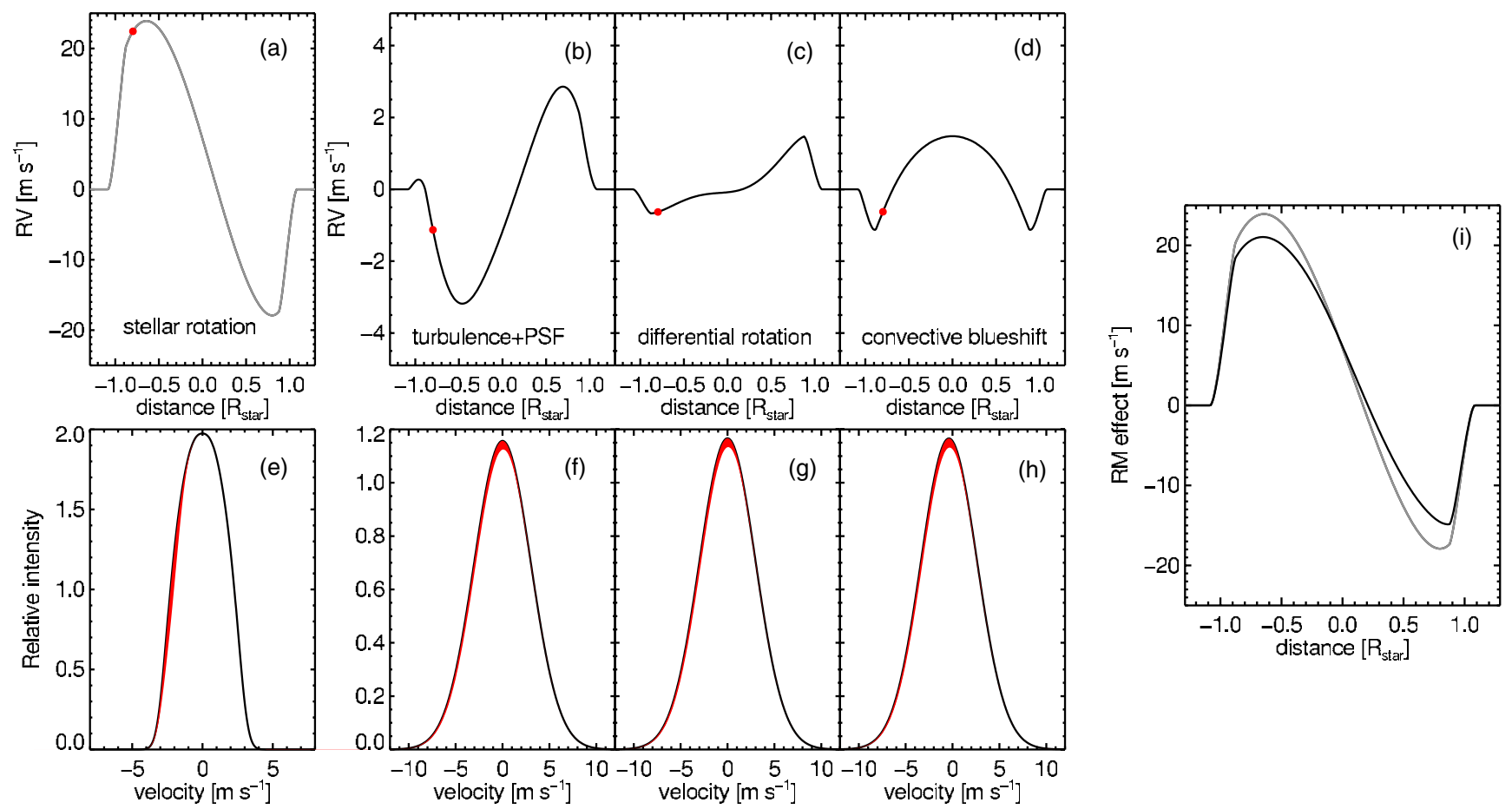

Figure 1. Illustration of the influence of rotation, PSF broadening and macroturbulence, differential rotation, and convective blueshift on the RM effect. (a) The effect of rotation. The chosen parameters are $\lambda=40^{\circ}, v \sin i_{\star}=3 \mathrm{~km} \mathrm{~s}^{-1}, R_{\mathrm{p}} / R_{\star}=0.12$, and an impact parameter of 0.2 . The $x$-coordinate is the planet's location relative to its position at inferior conjunction. (b) The effects of instrumental broadening $\left(2.2 \mathrm{~km} \mathrm{~s}^{-1}\right)$ and macroturbulence $\left(\zeta_{\mathrm{RT}}=3 \mathrm{~km} \mathrm{~s}{ }^{-1}\right)$. (c) The effect of solar-like differential rotation. (d) The effect of a solar-like convective blueshift. (e-h) The corresponding models for the stellar absorption lines. The red region corresponds to the light lost during the particular transit phase indicated by a red dot in the upper panel. For the lower panels the radius of the planet was doubled, for improved visibility of the missing velocity components. (i) The combined model including all aforementioned effects. The gray line is the model from panel (a).

(A color version of this figure is available in the online journal.)

while near the limb, the Doppler shifts are produced by motions along the stellar surface ("tangential" motions). The net effect is obtained by spatial integration over the visible hemisphere. Hirano et al. (2011c) provided a semi-analytic description of this effect, which we used in our model. The macroturbulent field is parameterized by $\zeta_{\mathrm{RT}}$, the average value of the radial and tangential velocities (assumed to be equal).

What value of $\zeta_{\mathrm{RT}}$ should be used to model the RM effect for a given system? Measuring macroturbulence from the line profile is challenging, as its measurement is correlated with the measurement of $v \sin i_{\star}$. In principle, the RM effect itself could be used to measure macroturbulence. However, for most of the systems analyzed here the data sets are not precise enough to allow $\zeta_{\mathrm{RT}}$ to be a free parameter. If the macroturbulence cannot be determined by the RM effect, one might think its value also does not matter for the analysis. However, as described below, we used prior information on $v \sin i_{\star}$ as a constraint on our model, and this information can only be properly incorporated if macroturbulence is taken into account. We therefore made use of the relation given by (Gray 1984, Equation (4)),

$$
\zeta_{\mathrm{RT}}=3.95 T_{\text {eff }}-19.25,
$$

where $\zeta_{\mathrm{RT}}$ is in $\mathrm{km} \mathrm{s}^{-1}$ and $T_{\text {eff }}$ in thousands of Kelvin. Gray (1984) gives an uncertainty of $0.2 \mathrm{~km} \mathrm{~s}^{-1}$ for this relationship between $T_{\text {eff }}$ and $\zeta_{\mathrm{RT}}$. We adopt a more conservative uncertainty estimate of $1.5 \mathrm{~km} \mathrm{~s}^{-1}$. We decided to use the relationship by Gray (1984) for macroturbulence instead of the relationship by Valenti \& Fischer (2005) because the latter authors derived the relationship between $T_{\text {eff }}$ and $\zeta_{\mathrm{RT}}$ for $\mathrm{F}$ stars by extrapolation from later spectral types, while Gray (1984) obtained macroturbulence values for hotter stars directly. Many of the stars in our sample have a $T_{\text {eff }}$ greater then $6000 \mathrm{~K}$.
Additional contributions to the line width come from collisional broadening, and Zeeman splitting, which are small compared to the effects described above. We account for them, at least approximately, by convolving the disk-integrated line profiles with a Lorentzian function of width $1 \mathrm{~km} \mathrm{~s}^{-1}$.

\subsection{Convective Blueshift}

Upward-flowing material in a convective cell is hotter and more luminous than downward-flowing material. This leads to a net blueshift in the disk-integrated light, known as the convective blueshift (CB). The CB is strongest in light received from the center of the disk and weaker near the stellar limb. Disk integration leads to an overall Doppler shift and an asymmetry in the stellar absorption lines. The overall Doppler shift is of order $1 \mathrm{~km} \mathrm{~s}^{-1}$, but this is unimportant for our purposes, as we are only interested in relative RVs. However, the motion of the transiting planet will cause time variations in the net $\mathrm{CB}$ of the exposed surface of the star. The time-varying component of the CB effect is of order $1 \mathrm{~m} \mathrm{~s}^{-1}$ for transits of late-type stars (Figure 1). This is a small effect but for completeness it was included in our model.

To describe the effect of the CB, we used a numerical model based on work by Shporer \& Brown (2011). For the stars in our sample with $T_{\text {eff }}<6000 \mathrm{~K}$ we assumed the net convective blueshift to be similar to that of the Sun: an effective RV of the photosphere of $500 \mathrm{~m} \mathrm{~s}^{-1}$. For hotter stars we assumed an effective RV of $1000 \mathrm{~m} \mathrm{~s}^{-1}$. The single exception to this rule was Gl 436, which is much cooler than the other stars $\left(T_{\text {eff }}=3350 \pm 300 \mathrm{~K}\right)$. For this system we assumed a smaller blueshift of $200 \mathrm{~m} \mathrm{~s}^{-1}$. In principle, one could estimate the overall CB for each system based on the observed line bisectors, 
but we did not pursue this approach because our first-order analysis revealed that the $\mathrm{CB}$ effect is relatively unimportant.

\subsection{Differential Rotation}

For this study, we neglected the possible influence of differential surface rotation on the RM effect. Albrecht et al. (2012) concluded that modeling differential rotation is justified only if the transit chord spans a wide range of stellar latitudes, the RM effect is detected with a very high signal-to-noise ratio $(\mathrm{S} / \mathrm{N})$, and excellent prior constraints are available for the limb darkening (not only as it affects the continuum but also the absorption lines). These conditions are not met simultaneously for any of the systems in our sample. See Albrecht et al. (2012) for further discussion and an application of a model with differential rotation to the WASP-7 system.

\subsection{RV Measurements During Transits}

A model which aims to simulate RV measurements made during transits needs to take into account that the measured RVs represent the output of a Doppler-measuring code. These codes are complex but in essence they locate the peak of a crosscorrelation between a stellar template spectrum obtained outside transit and a spectrum observed during transit. Depending on the system parameters these RVs can significantly differ from $\mathrm{RV}$ s representing the first moment of the absorption line. The later are often used in RM work potentially leading to systematic errors in the derived parameters (Hirano et al. 2010, 2011c).

Hirano et al. (2011c) presented a semi-analytic description of the shift in the cross-correlation peak as a function of the transit parameters, including the dependence of the RVs on the stellar rotation velocity and obliquity, the microturbulent and macroturbulent velocities, the differential rotation profile, and the PSF of the spectrograph. We used this model after extending it to include the convective blueshift. We also tested the results of the code by Hirano et al. (2011c) with the fully numerical disk-integration code of Albrecht et al. (2007), including the same physical effects, finding good agreement. Figure 1 shows the influence of the effects discussed in this and the previous sections on the stellar absorption lines.

\subsection{Other RV Variation Sources}

In addition to the RM effect we must also model the changes in RV due to the star's orbital motion. Over the course of the transit night, this motion can usually be represented as a linear function of time and parameterized by a constant acceleration. For ease of comparison with existing orbital solutions, the outof-transit velocity trend may also be parameterized by the orbital velocity semi-amplitude $\left(K_{\star}\right)$, for fixed values of the other orbital parameters (period $P$, eccentricity $e$, and argument of pericenter $\omega$ ).

The question arises whether to allow $K_{\star}$ to be a completely free parameter in our analysis or whether to use a prior constraint based on a previously published orbital solution. The answer is not obvious because stars exhibit intrinsic RV noise that may have different amplitudes on different timescales, ranging from a few minutes to days. Short-timescale noise will simply degrade our measurement accuracy, but noise on timescales longer than $\sim 6 \mathrm{hr}$ (such as the noise produced by rotating starspots) will introduce trends in the RV signal over the course of the transit night. Without a specific model for the frequency content of the intrinsic RV noise, it is difficult to combine the data obtained sporadically over days or months with the data obtained with

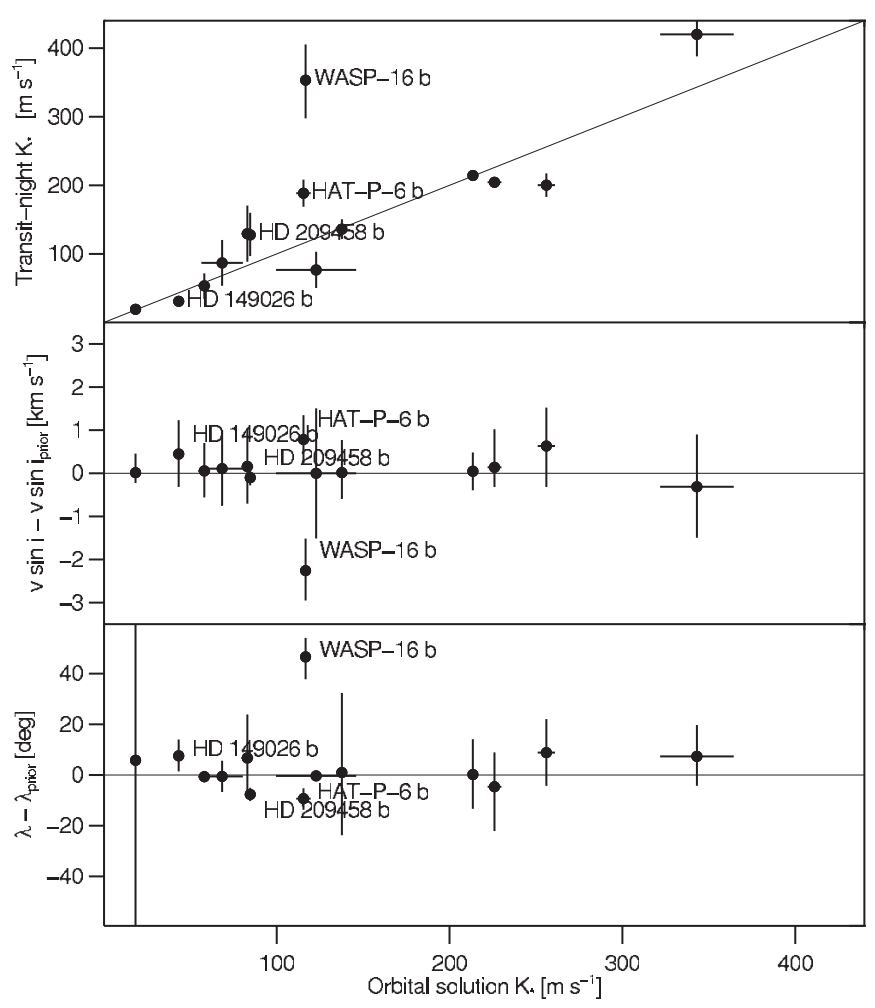

Figure 2. Comparison between results with and without a prior on $K_{\star}$. Upper panel: measurements of $K_{\star}$ based on the transit-night velocity gradient versus previously reported measurements of $K_{\star}$ from orbital solutions based on RV data obtained over months or years. (The three systems with the largest $K_{\star}$ are omitted to permit the other systems to be viewed more clearly.) While for many systems the results are consistent, there are a number of systems showing disagreement, possibly due to stellar RV noise with a timescale of hours to days. Middle panel: difference between $v \sin i_{\star}$ values obtained with and without a constraint on $K_{\star}$ based on the orbital solution. Lower panel: same as middle panel, but for $\lambda$.

much finer time sampling on a single night. Specifically, if we would choose to constrain $K_{\star}$ (and therefore the transit-night velocity gradient) based on the orbital solution, there is a risk of introducing a bias in our results because of actual transitnight velocity gradient could be affected by starspot variability or other intrinsic RV noise with timescales longer than a few hours (Bouchy et al. 2008; Albrecht et al. 2011b, 2012).

One might proceed by downweighting the constraint on $K_{\star}$ by some amount deemed to be appropriate, or fitting all of the RV data using a frequency-dependent noise model. For this work we placed a high premium on simplicity and homogeneity, and decided to decouple the transit-night data from the rest of the data. We allowed $K_{\star}$ to be a completely free parameter and determine the velocity gradient from the transit-night data alone. This was done for all the systems in our sample, even those where we find no obvious sign of intrinsic RV noise. This leads to larger uncertainties, and for some systems we find wider confidence intervals for $\lambda$ than other researchers using similar data or even noisier data. While in some systems this might be an unnecessary precaution, the stars' characteristics are in general not well enough known to make a principled case-bycase decision. Table 4 gives the values of $K_{\star}$ from published orbital solutions, along with the values of $K_{\star}$ derived from our model which was fitted to the transit-night data.

For those readers who do not agree with our reasoning on this point or who are simply curious about the effect of imposing a prior on $K_{\star}$, Table 4 and Figure 2 also provide the results for 
Table 3

Prior Knowledge

\begin{tabular}{|c|c|c|c|c|c|c|c|c|c|}
\hline System & $\begin{array}{c}\text { Jitter } \\
\left(\mathrm{m} \mathrm{s}^{-1}\right)\end{array}$ & $\begin{array}{c}T_{c}-2400000 \\
\left(\mathrm{BJD}_{\mathrm{TDB}}\right)\end{array}$ & $\begin{array}{l}T_{41} \\
(\mathrm{hr})\end{array}$ & $\begin{array}{l}T_{21} \\
(\mathrm{hr})\end{array}$ & $R_{\mathrm{p}} / R_{\star}$ & $u 1+u 2$ & $\begin{array}{c}\zeta_{\mathrm{RT}} \\
\left(\mathrm{km} \mathrm{s}^{-1}\right)\end{array}$ & $\begin{array}{c}v \sin i_{\star} \\
\left(\mathrm{km} \mathrm{s}^{-1}\right)\end{array}$ & Referen \\
\hline AT-P-6 & 70 & $54035.67652 \pm 0.00196$ & $3.506 \pm 0.041$ & $0.451 \pm 0.053$ & $934 \pm 0.0005$ & $0.67 \pm 0.10$ & $5.52 \pm 1.50$ & $8.7 \pm 1.5$ & 1 \\
\hline HAT-P-7 & 6.0 & $55401.91904 \pm 0.00002$ & $3.941 \pm 0.002$ & $73 \pm 0.004$ & $7777 \pm 0.0001$ & 10 & .50 & $8=$ & 2 \\
\hline HAT-P-24 & 12.0 & $55216.97667 \pm 0.00071$ & $3.694 \pm 0.019$ & $0.338 \pm 0.029$ & $.0970 \pm$ & $0.70 \pm$ & $5.22 \pm 1.50$ & $10.0=$ & 4 \\
\hline HAT-P-32 & 42.0 & $54420.44637 \pm 0.00069$ & $3.108 \pm 0.007$ & $0.413 \pm 0.010$ & $-\sigma^{2}$ & $0.69 \pm$ & & & 5 \\
\hline HAT-P-34 & 25.0 & $55431.59629 \pm 0.00$ & $3.492 \pm 0$ & 0.290 & & & & & 6 \\
\hline WAS & & 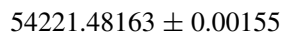 & $1+$ & 4 & & & & 11. & 10 \\
\hline WAS & 20.0 & 1 & 1. & 4 & 4 & 10 & & & 11,12 \\
\hline WAS & 10.0 & 75 & 2. & 5 & 7 & 0. & 4 & 2.4 & 13,14 \\
\hline WASP-31 & 6.0 & 09.7 & $0.110 \pm$ & $29 \pm$ & 11 & 0.6 & 50 & $7.9 \pm 1.5$ & 15 \\
\hline Gl 436 & 1.5 & $54235.83624 \pm 0.01221$ & $1.012 \pm 0.010$ & $0.213 \pm 0.028$ & $0.0825 \pm 0.0078$ & $0.87 \pm 0.10$ & $0.56 \pm 1.50$ & $0+5$ & This we \\
\hline epler-8 & 10.0 & $55781.90611 \pm 0.00032$ & $3.257 \pm 0.010$ & $0.557 \pm 0.029$ & $0.0948 \pm 0.0006$ & $0.71 \pm 0.10$ & $4.97 \pm 1.50$ & $10.5 \pm 1.5$ & 16 \\
\hline
\end{tabular}

Reference. (1) Noyes et al. 2008; (2) Pál et al. 2008; (3) Buchhave et al. 2010; (4) Kipping et al. 2010; (5) Hartman et al. 2011; (6) Bakos et al. 2012; (7) Maciejewski et al. 2011; (8) Hebb et al. 2009; (9) Lister et al. 2009; (10) Hellier et al. 2009; (11) Hebb et al. 2010; (12) Hellier et al. 2011; (13) Smalley et al. 2010; (14) Anderson et al. 2011a; (15) Anderson et al. 2011b; (16) Jenkins et al. 2010; (17) Pál et al. 2010; (18) Carter et al. 2009; (19) Sato et al. 2005; (20) Laughlin et al. 2005.

$\lambda$ and $v \sin i_{\star}$ obtained by imposing a prior on $K_{\star}$ based on the orbital solution.

\subsection{Quantitative Analysis}

Having described the model, we now describe the procedure for parameter estimation. For each observed transit, we calculated the expected time of inferior conjunction $\left(T_{\mathrm{c}}\right)$ based on the published ephemerides. This predicted time was used as a prior in our analysis of the transit-night RV data. The out-oftransit variation was parameterized by the semi-amplitude of the projected stellar reflex motion $\left(K_{\star}\right)$ and an arbitrary additive constant velocity $(\gamma)$.

The transit geometry was described by the following parameters: the radius of the star in units of the orbital semi-major axis $\left(R_{\star} / a\right)$, the radius of the planet in units of the stellar radius $\left(R_{\mathrm{p}} / R_{\star}\right)$, and the cosine of the orbital inclination $\left(\cos i_{\mathrm{o}}\right)$. We imposed Gaussian priors on $R_{\mathrm{p}} / R_{\star}$, the total transit duration from first to last contact $\left(T_{41}\right)$, and the ingress duration $\left(T_{21}\right)$, based on previously reported photometric analyses. By choosing those particular parameters we minimized the correlations among their uncertainties (Carter et al. 2008), consistent with our treatment of the priors as independent Gaussian functions. However, small correlations do exist and affect $T_{21}$ most strongly. In the interest of homogeneity we took the simple and conservative approach of doubling the reported uncertainty in $T_{21}$ for all systems. Table 3 specifies all the priors that were used in practice, including the increased uncertainty in $T_{21}$.

For systems where values for $T_{41}$ or $T_{21}$ were not previously reported in the literature we obtained a transit light curve and derived those parameters ourselves, using the transit model of Mandel \& Agol (2002). The uncertainty intervals in $R_{\mathrm{p}} / R_{\star}, T_{41}$, and $T_{21}$ were then used as Gaussian priors in the RV analysis in the same way as for all the other systems, including the doubled uncertainty in $T_{21}$.

We assumed a quadratic limb-darkening law with parameters $u_{1 \mathrm{RM}}$ and $u_{2 \mathrm{RM}}$ selected from the tables by Claret (2000) for the Johnson $V$ band (similar to the iodine spectral region). We used the "jktld" 11 tool to query the ATLAS models for all systems except Gl 436, for which we used the tables based on the Phoenix code. We placed a Gaussian prior on $u_{1 \mathrm{RM}}+u_{2 \mathrm{RM}}$ with a width of 0.1 . The difference $u_{1 \mathrm{RM}}-u_{2 \mathrm{RM}}$ was held fixed at the tabulated value, since this combination is weakly constrained by the data and has minimal effect on the other parameters.

Because $\lambda$ and $v \sin i_{\star}$ have correlated uncertainties, we used the fitting parameters $\sqrt{v \sin i_{\star}} \cos \lambda$ and $\sqrt{v \sin i_{\star}} \sin \lambda$. A final constraint used in our fitting statistic is the prior knowledge concerning the projected rotation speed, which was measured for all systems in our sample. Here we adopted a minimum uncertainty of $1.5 \mathrm{~km} \mathrm{~s}^{-1}$ even when the reported uncertainty was lower. The $v \sin i_{\star}$ measurements in our sample were conducted by different researchers using different approaches and it is not clear that the scales for the different approaches are identical, particularly for the very challenging cases of low projected rotation speeds. Furthermore, we expect that our lack of knowledge on differential rotation, macroturbulence, and spectroscopic limb darkening introduces systematic errors at that level. All constraints used in our fits are listed in Table 3.

Finally, we must specify the width of a Gaussian function representing the contribution to the line width due to both microturbulence and the PSF of the spectrograph. The influence of both can be approximated by a convolution with a single Gaussian, despite their difference in origin (Hirano et al. 2011c). Assuming a value of $2 \mathrm{~km} \mathrm{~s}^{-1}$ for the microturbulence parameter and $2.2 \mathrm{~km} \mathrm{~s}^{-1}$ for the PSF width at $5500 \AA$, we obtain a $\sigma$ of $3 \mathrm{~km} \mathrm{~s}^{-1}$ for this purpose. The results for $\lambda$ and $v \sin i_{\star}$ do not depend strongly on the values of these parameters.

To derive confidence intervals for the parameters we used the Markov Chain Monte Carlo algorithm. Before starting the chain we also added "stellar jitter" terms in quadrature to the internally estimated uncertainty of the RVs to obtain a reduced $\chi^{2}$ close to unity. In making this step we assumed that the uncertainties in the RV measurements within a given night are uncorrelated 11 This code is kindly made available by J. Southworth:
http://www.astro.keele.ac.uk/jkt/codes/jktld.html. 
Table 4

Results for Rotation Parameters

\begin{tabular}{|c|c|c|c|c|c|c|c|c|c|}
\hline \multicolumn{6}{|c|}{ Final Results } & \multicolumn{4}{|c|}{ With Prior on $K_{\star}{ }^{\mathrm{a}}$} \\
\hline System & $\begin{array}{c}\sqrt{v \sin i_{\star}} \sin \lambda \\
\left(\mathrm{km} \mathrm{s}^{-1}\right)\end{array}$ & $\begin{array}{c}\sqrt{v \sin i_{\star}} \cos \lambda \\
\left(\mathrm{km} \mathrm{s}^{-1}\right)\end{array}$ & $\begin{array}{c}v \sin i_{\star} \\
\left(\mathrm{km} \mathrm{s}^{-1}\right)\end{array}$ & $\begin{array}{c}\lambda \\
\left({ }^{\circ}\right)\end{array}$ & $\begin{array}{l}K_{\star} \text { transit } \\
\left(\mathrm{km} \mathrm{s}^{-1}\right)\end{array}$ & $\begin{array}{c}K_{\star} \text { literature } \\
\left(\mathrm{km} \mathrm{s}^{-1}\right)\end{array}$ & $\begin{array}{c}v \sin i_{\star} \\
\left(\mathrm{km} \mathrm{s}^{-1}\right)\end{array}$ & $\begin{array}{c}\lambda \\
\left({ }^{\circ}\right)\end{array}$ & Reference $^{b}$ \\
\hline HAT-P-6 & $0.702 \pm 0.269$ & $-2.689 \pm 0.131$ & $7.8 \pm 0.6$ & $165 \pm 6$ & $188 \pm 20$ & $115.5 \pm 4.2$ & $7.0 \pm 0.6$ & $175 \pm 4$ & 1 \\
\hline HAT-P-7 & $0.695 \pm 0.361$ & $-1.476 \pm 0.196$ & $2.7 \pm 0.5$ & $155 \pm 37^{\mathrm{c}}$ & $214 \pm 5$ & $213.5 \pm 1.9$ & $2.7 \pm 0.4$ & $155 \pm 14$ & 2 \\
\hline HAT-P-16 & $-0.051 \pm 1.413$ & $1.327 \pm 0.364$ & $3.1 \pm 1.0$ & $-2_{-46}^{+55}$ & $536 \pm 60$ & $531.1 \pm 2.8$ & $2.7 \pm 0.8$ & $-6 \pm 37$ & 3 \\
\hline HAT-P-24 & $1.158 \pm 0.851$ & $3.087 \pm 0.297$ & $11.2 \pm 0.9$ & $20 \pm 16$ & $129 \pm 41$ & $83 \pm 3.4$ & $11.0 \pm 0.9$ & $14 \pm 16$ & 4 \\
\hline HAT-P-32 & $4.517 \pm 0.168$ & $0.396 \pm 0.117$ & $20.6 \pm 1.5$ & $85.0 \pm 1.5$ & $77 \pm 26$ & $122.8 \pm 23.2$ & $20.6 \pm 1.5$ & $85.3 \pm 1.5$ & 5 \\
\hline HAT-P-34 & $-0.004 \pm 1.164$ & $4.822 \pm 0.179$ & $24.3 \pm 1.2$ & $0 \pm 14$ & $421 \pm 32$ & $343.1 \pm 21.3$ & $24.6 \pm 1.2$ & $-7 \pm 12$ & 6 \\
\hline WASP-12 & $1.044 \pm 0.399$ & $0.640 \pm 0.210$ & $1.6_{-0.4}^{+0.8}$ & $59_{-20}^{+15}$ & $204.4 \pm 2.4$ & $226 \pm 4$ & $1.4_{-0.5}^{+0.9}$ & $63_{-21}^{+14}$ & 7 \\
\hline WASP-16 & $0.324 \pm 0.617$ & $1.674 \pm 0.376$ & $3.2 \pm 0.9$ & $11_{-19}^{+26}$ & $353 \pm 54$ & $116.7 \pm 2.4$ & $5.4 \pm 0.7$ & $-36_{-10}^{+7}$ & 8 \\
\hline WASP-18 & $0.774 \pm 0.421$ & $3.234 \pm 0.126$ & $11.2 \pm 0.6$ & $13 \pm 7$ & $1768 \pm 5$ & $1818.3 \pm 8.0$ & $10.9 \pm 0.6$ & $12.6 \pm 7$ & 9 \\
\hline WASP-19 & $0.552 \pm 0.371$ & $1.984 \pm 0.241$ & $4.4 \pm 0.9$ & $15 \pm 11$ & $200 \pm 17$ & $256 \pm 5$ & $3.7 \pm 0.9$ & $7 \pm 13$ & 10 \\
\hline WASP-26 & $-0.803 \pm 0.722$ & $1.117 \pm 0.304$ & $2.2 \pm 0.7$ & $-34_{-26}^{+36}$ & $137 \pm 15$ & $137.7 \pm 1.5$ & $2.6 \pm 0.9$ & $-42_{-25}^{+34}$ & 11 \\
\hline WASP-31 & $0.076 \pm 0.130$ & $2.605 \pm 0.121$ & $6.8 \pm 0.6$ & $-6 \pm 6$ & $53 \pm 19$ & $58.1 \pm 3.4$ & $7.2 \pm 0.6$ & $-6 \pm 3$ & 12 \\
\hline Gl 436 & $-0.081 \pm 0.357$ & $-0.192 \pm 0.584$ & $<0.4$ & $\ldots$ & $19 \pm 3$ & $18.34 \pm 0.52$ & $<0.4$ & $\ldots$ & 13 \\
\hline Kepler-8 & $0.255 \pm 0.353$ & $2.960 \pm 0.179$ & $8.9 \pm 1.0$ & $5 \pm 7$ & $74 \pm 37$ & $68.4 \pm 12.0$ & $8.8 \pm 0.9$ & $-5 \pm 6$ & 14 \\
\hline HAT-P-2 & $0.697 \pm 0.341$ & $4.349 \pm 0.101$ & $19.5 \pm 1.4$ & $9 \pm 10$ & $808 \pm 9$ & $983.9 \pm 17.2$ & $19.2 \pm 0.7$ & $9 \pm 4$ & 15 \\
\hline HD 149026 & $0.558 \pm 0.339$ & $2.706 \pm 0.157$ & $7.7 \pm 0.8$ & $12 \pm 7$ & $31 \pm 6$ & $43.3 \pm 1.2$ & $7.2 \pm 0.7$ & $4 \pm 6$ & 16 \\
\hline HD 209458 & $-0.167 \pm 0.237$ & $2.084 \pm 0.056$ & $4.4 \pm 0.2$ & $-5 \pm 7$ & $128 \pm 32$ & $84.67 \pm 0.70$ & $4.5 \pm 0.2$ & $3 \pm 3$ & 17 \\
\hline
\end{tabular}

Notes.

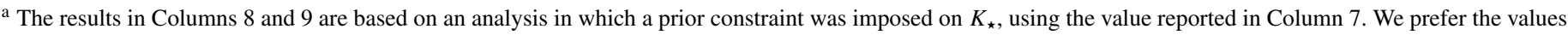
in Columns 4 and 5 for reasons described in Section 3.5.

b Reference for the value of $K_{\star}$ in Column 7 .

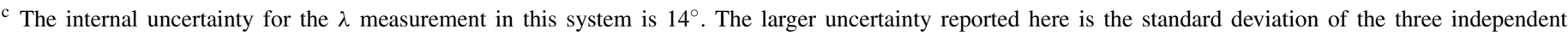
measurements, as discussed in Section 4.2.

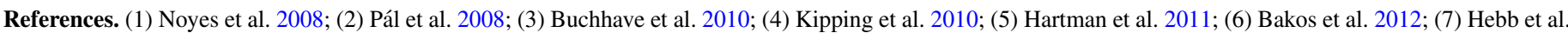

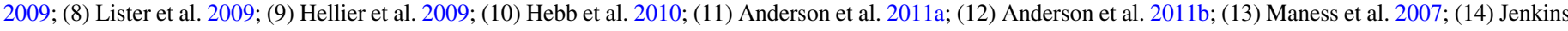
et al. 2010; (15) Pál et al. 2010; (16) Sato et al. 2005; (17) Torres et al. 2008.

and Gaussian. Table 2 reports the original, internally estimated uncertainties without any jitter term. The added terms are listed for each system in Table 3.

The results reported in Table 4 are the median values of the posterior distribution. The quoted uncertainty intervals represent the range that excludes $15.85 \%$ of the values on each side of the posterior distribution and encompass $68.3 \%$ of the probability.

\section{NOTES ON INDIVIDUAL SYSTEMS}

In this section, we report briefly on each of the 17 systems in our sample (14 new observations and three re-analyses of previously published data). We mention whenever we deviated from the general approach described above. We also discuss briefly the values for $v \sin i_{\star}$ and $\lambda$ that were obtained. For cases in which $\lambda$ has been previously reported in the literature, we compare the new value with the previous value.

\subsection{HAT-P-6}

The discovery of the hot Jupiter HAT-P-6 b was reported by Noyes et al. (2008). We obtained 42 HIRES spectra over a $5 \mathrm{hr}$ period covering the transit, which occurred on the night of 2010 November $5 / 6$. Although little data could be obtained before ingress, the observations continued for $1.5 \mathrm{hr}$ after the $3.5 \mathrm{hr}$ transit. All the RVs are shown in the left panels of Figure 3 along with the best-fitting model. The RV curve immediately reveals a retrograde orbit. The right panels show the results in the $\lambda-v \sin i_{\star}$ plane. To obtain these we used the HIRES RVs together with the prior information from Noyes et al. (2008) which are reported in Table 3 . We find $\lambda=165^{\circ} \pm 6^{\circ}$ and $v \sin i_{\star}=7.8 \pm 0.6 \mathrm{~km} \mathrm{~s}^{-1}$. With this we confirm, with higher precision, the measurement presented by Hébrard et al. (2011a). They found $\lambda=166^{\circ} \pm 10^{\circ}$ and $v \sin i_{\star}=7.5 \pm 1.6 \mathrm{~km} \mathrm{~s}^{-1}$.

When the model was rerun with a prior constraint on $K_{\star}$, we obtained $\lambda=175^{\circ} \pm 4^{\circ}$, in disagreement with the priorfree result and with the previously reported value. (See Table 4 and Figure 2.) Apparently, the star exhibits RV noise that is correlated on the timescale of at least a few hours.

\subsection{HAT-P-7}

Pál et al. (2008) reported the discovery of HAT-P-7b. This was one of the first systems for which a significant misalignment between the stellar spin and orbital angular momentum was discovered in a star-planet system (Winn et al. 2009; Narita et al. 2009 b). Both research groups reported an apparently retrograde orbit. In fact this star is likely to be viewed nearly pole-on, judging from its unusually low $v \sin i_{\star}$ (Winn et al. 2009; Schlaufman 2010), but with nearly antiparallel sky projections of the orbital and stellar rotation vectors.

Interestingly, the two independent results for $\lambda$ were in disagreement, with Winn et al. (2009) reporting $\lambda$ of $182.5 \pm 9^{\circ}$ and $v \sin i_{\star}=4.9_{-0.9}^{+1.2} \mathrm{~km} \mathrm{~s}^{-1}$, and Narita et al. (2009b) reporting $\lambda=-132.6_{-16.3}^{+10.5}$ (equivalent to $\lambda=227.4_{-16.3^{\circ}}^{+10.5}$ ) and $v \sin i_{\star}=2.3_{-0.5}^{+0.6} \mathrm{~km} \mathrm{~s}^{-1}$. Both groups used the same instrument (High Dispersion Spectrograph). With HIRES we reobserved the system before, during, and after a planetary transit occurring during the night 2010 July 23/24. The RVs are displayed in Figure 4. While the RM effect is clearly detected, the $\mathrm{S} / \mathrm{N}$ of the detection is low enough that an analysis of the RM effect would benefit from a very precise prediction for the 

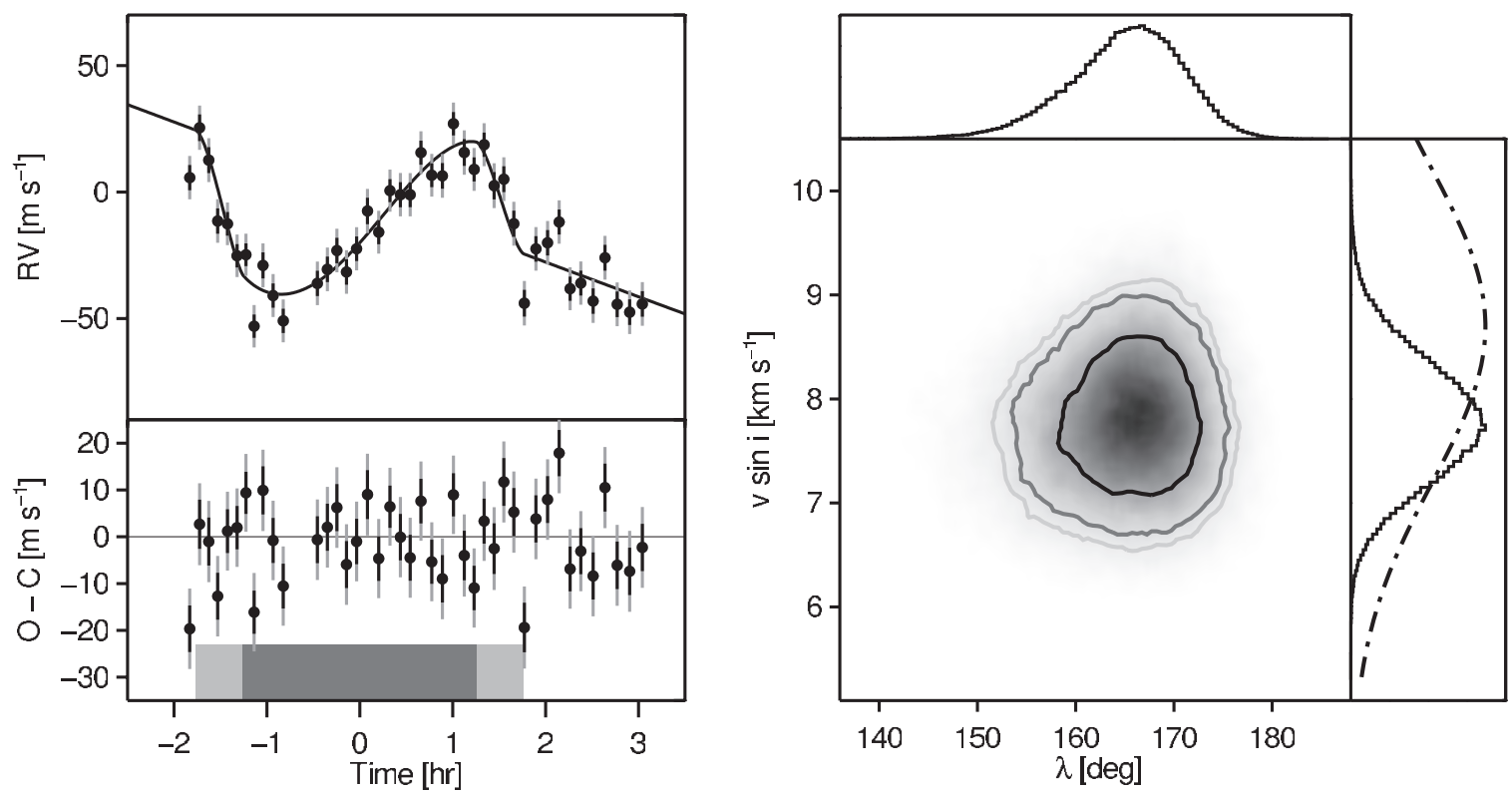

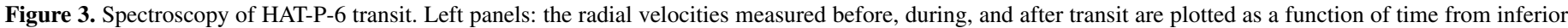

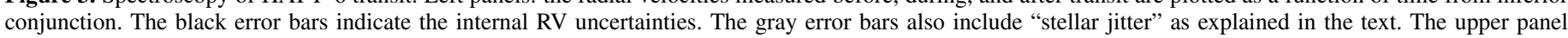

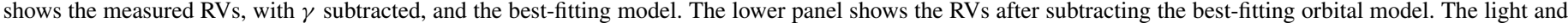

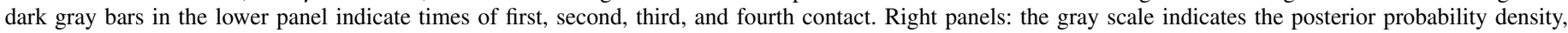

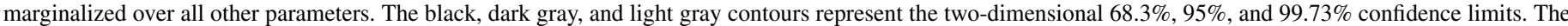
one-dimensional marginalized distributions are shown on the sides of the contour plot. The dash-dotted line shows the prior applied to $v$ sin $i_{\star}$.
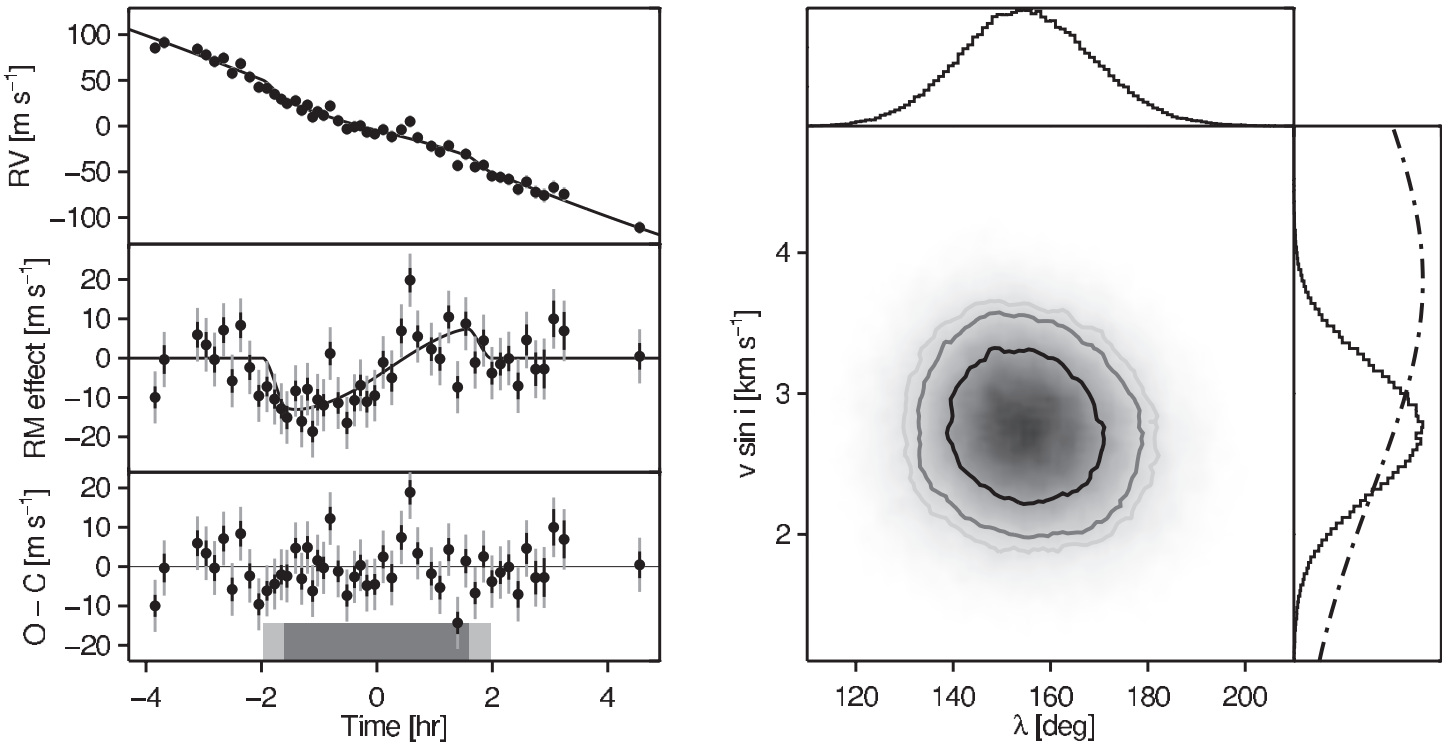

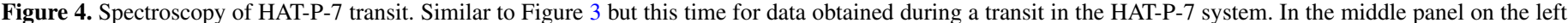
side, the orbital contribution to the observed RVs has been subtracted, isolating the RM effect.

transit midpoint. For this reason we downloaded the Quarter 6 Kepler light curve for this system, covering the epoch of our spectroscopic transit observations. The analysis of transit light curves is described in Section 3.6 and the derived photometric priors are given in Table 3 . We obtain a $\lambda$ of $155^{\circ} \pm 14^{\circ}$ and a $v \sin i_{\star}=2.7 \pm 0.5 \mathrm{~km} \mathrm{~s}^{-1}$.

While the $v \sin i_{\star}$ is intermediate between the two values reported previously, and the finding of a retrograde orbit is confirmed, the value we obtained for $\lambda$ does not agree with either of the previously reported values. What can have caused the disagreement between the three results? While we cannot give a definite answer we suspect that two characteristics of this system might have been important. $K_{\star}$ is 10 times larger than the amplitude of the RM effect making a clean separation of the RM effect from the orbital RV challenging. In addition, the $\mathrm{RM}$ amplitude is with $\sim 20 \mathrm{~m} \mathrm{~s}^{-1}$ only a few times larger than the typical uncertainty in the RV measurements. Albrecht et al. (2011a) argued that for low $\mathrm{S} / \mathrm{N}$ detections and for $\lambda$ near $0^{\circ}$ or $180^{\circ}$ the uncertainty in $\lambda$ can be underestimated. However, we cannot rule out the possibility that our model is missing some aspect of stellar astrophysics, perhaps one that is most apparent for nearly pole-on systems. The HAT-P-7 system is the only system in our sample where there exists a significant disagreement between several independent measures of $\lambda$.

Since the discrepancies are unresolved, and to avoid overinterpretation of the results in the subsequent discussion, the uncertainty reported in Table 4 is the standard deviation $\left(37^{\circ}\right)$ of the three independent measurements. 

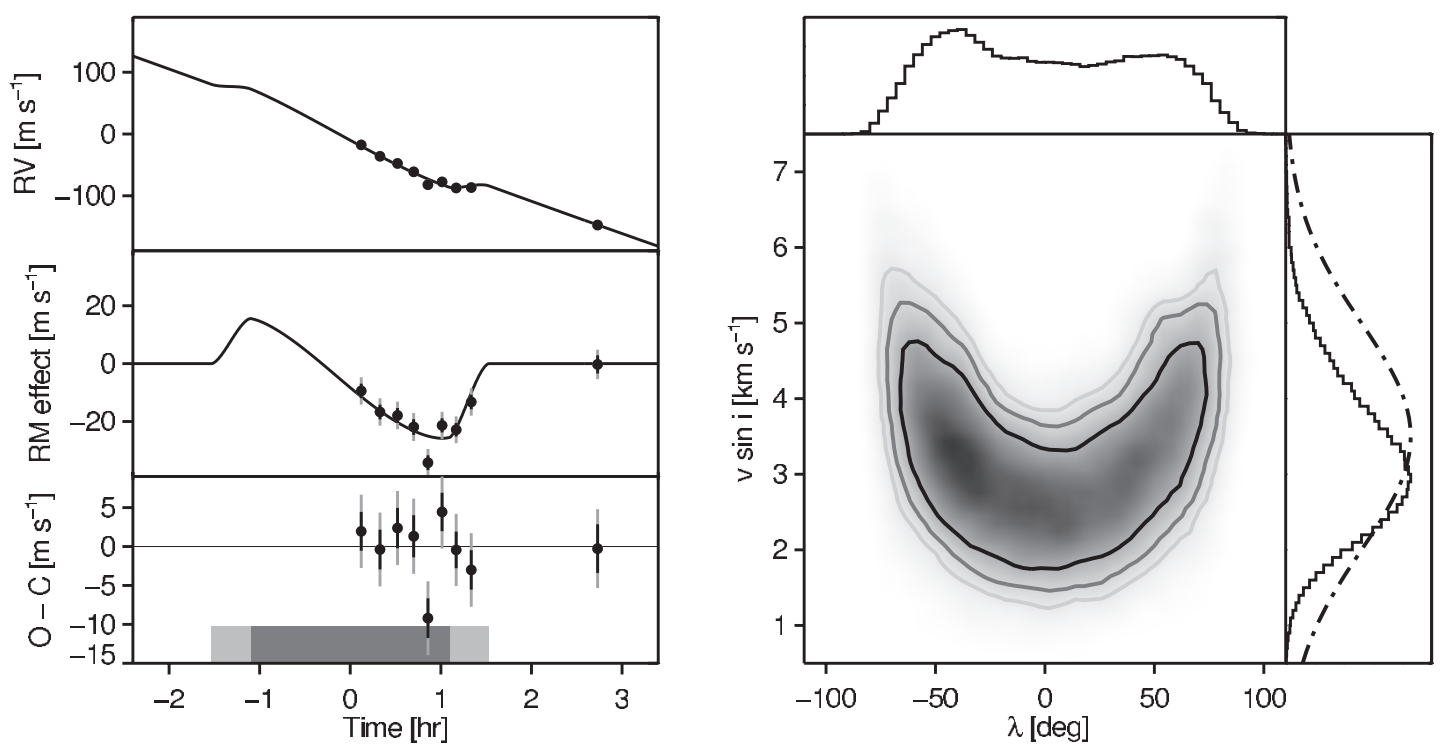

Figure 5. Spectroscopy of HAT-P-16 transit. Similar to Figure 4 but this time for data obtained during a transit in the HAT-P-16 system.
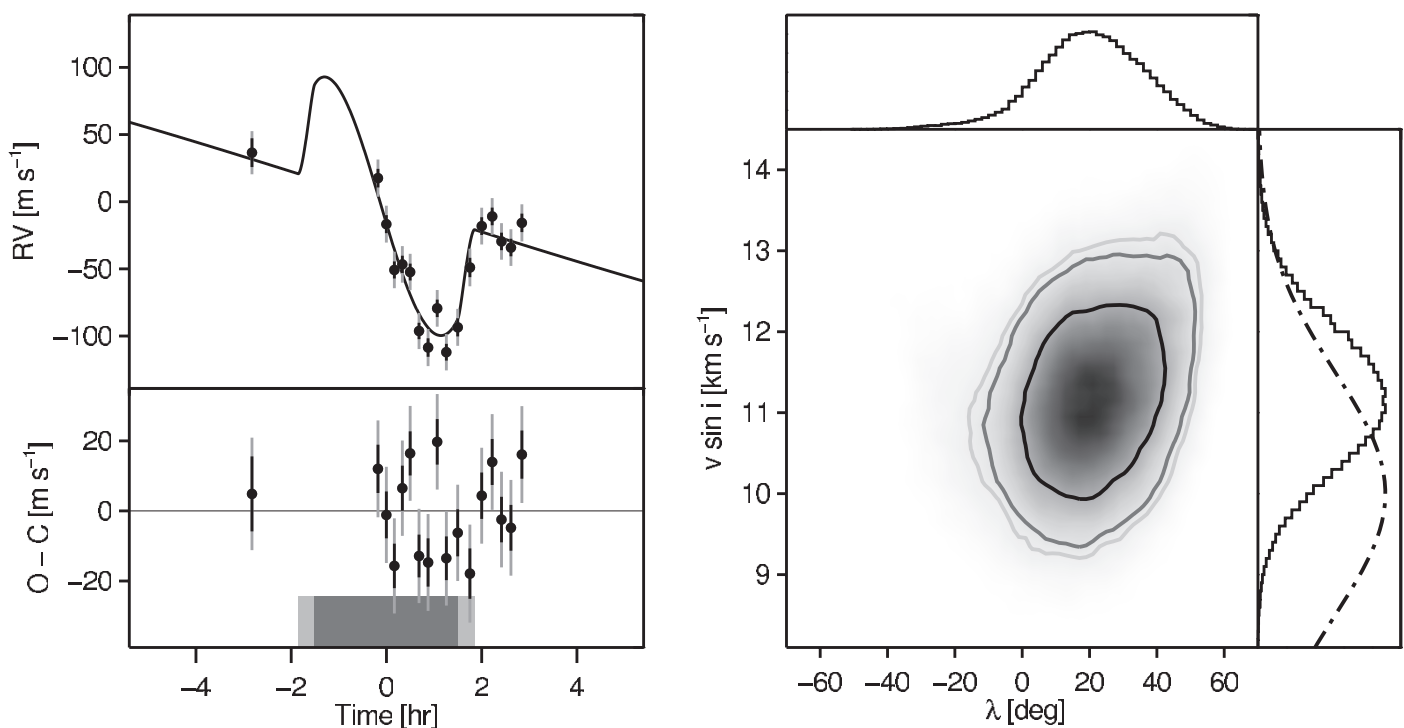

Figure 6. Spectroscopy of HAT-P-24 transit. Similar to Figure 3 but this time for data obtained during a transit in the HAT-P-24 system.

\subsection{HAT-P-16}

The existence of HAT-P-16b was announced by Buchhave et al. (2010). We observed this northern system with the Keck I telescope during the night 2010 December 26/27. Due to bad weather we were only able to observe the second half of the transit (Figure 5). No data before the transit were obtained. Using these RVs together with the photometric and $v \sin i_{\star}$ $\left(3.5 \pm 1.5 \mathrm{~km} \mathrm{~s}^{-1}\right)$ priors from Buchhave et al. (2010) we found that HAT-P-16 b orbits its host star on a prograde orbit $\left(\lambda=2_{-46}^{+55^{\circ}}\right)$. While the data are consistent with good alignment, little more can be learned. Concerning $v \sin i_{\star}$ we found a value of $3.1 \pm 1.0 \mathrm{~km} \mathrm{~s}^{-1}$, consistent with the prior. Moutou et al. (2011) found a projected obliquity of $10^{\circ} \pm 16^{\circ}$ for this system. In the subsequent discussion of Section 5, we adopt their value.

\subsection{HAT-P-24}

The 24th HATNet planet was announced by Kipping et al. (2010). We obtained HIRES spectra of this system during the night 2010 September $27 / 28$. As was the case for HAT-P-16, the observations were interrupted by bad weather, but at least for HAT-P-24 we obtained one pre-ingress data point (see Figure 6). This is why the result, $\lambda=20^{\circ} \pm 16^{\circ}$, is much more precise than for HAT-P-16. Nevertheless with the current data set is it not possible to exclude a small misalignment.

\subsection{HAT-P-32}

HAT-P-32 b was detected around a star which displays RV jitter on the order of $80 \mathrm{~m} \mathrm{~s}^{-1}$ (Hartman et al. 2011). Therefore the $\mathrm{S} / \mathrm{N}$ of the RM effect detection in our data set, obtained on 2011 December 5/6, is relatively low (see Figure 7). Nevertheless this is the system in our sample for which we obtain the highest precision in our measurement of the projected obliquity $\left(\lambda=85^{\circ} \pm 1.5\right)$. This curious fact is the consequence of the system having a low-impact parameter (0.12) and at the same time presenting an asymmetric RM curve.

For systems with low-impact parameters, even a strong misalignment will lead mostly to a reduction of the amplitude of the RM effect, with hardly any asymmetry in the RM curve. (e.g., Gaudi \& Winn 2007; Albrecht et al. 2011b). Only a narrow 

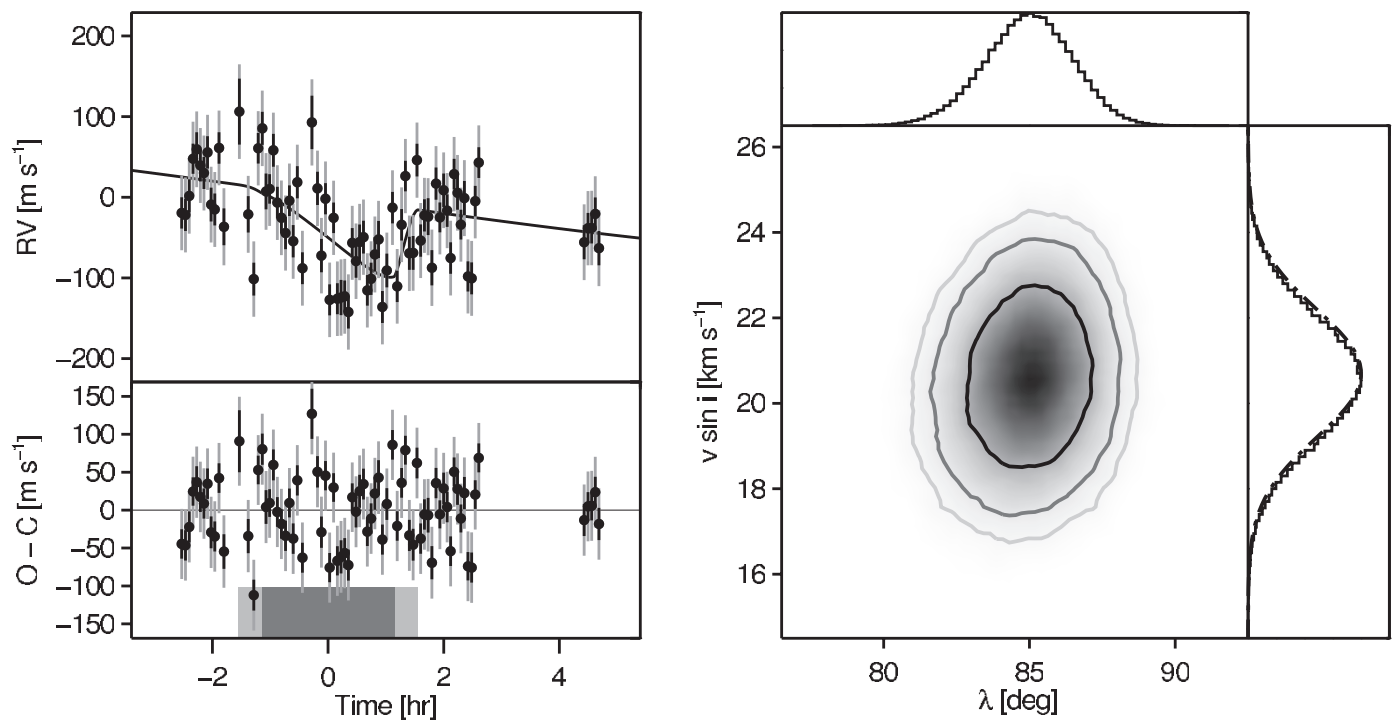

Figure 7. Spectroscopy of HAT-P-32 transit. Similar to Figure 3 but this time for data obtained during a transit in the HAT-P-32 system.
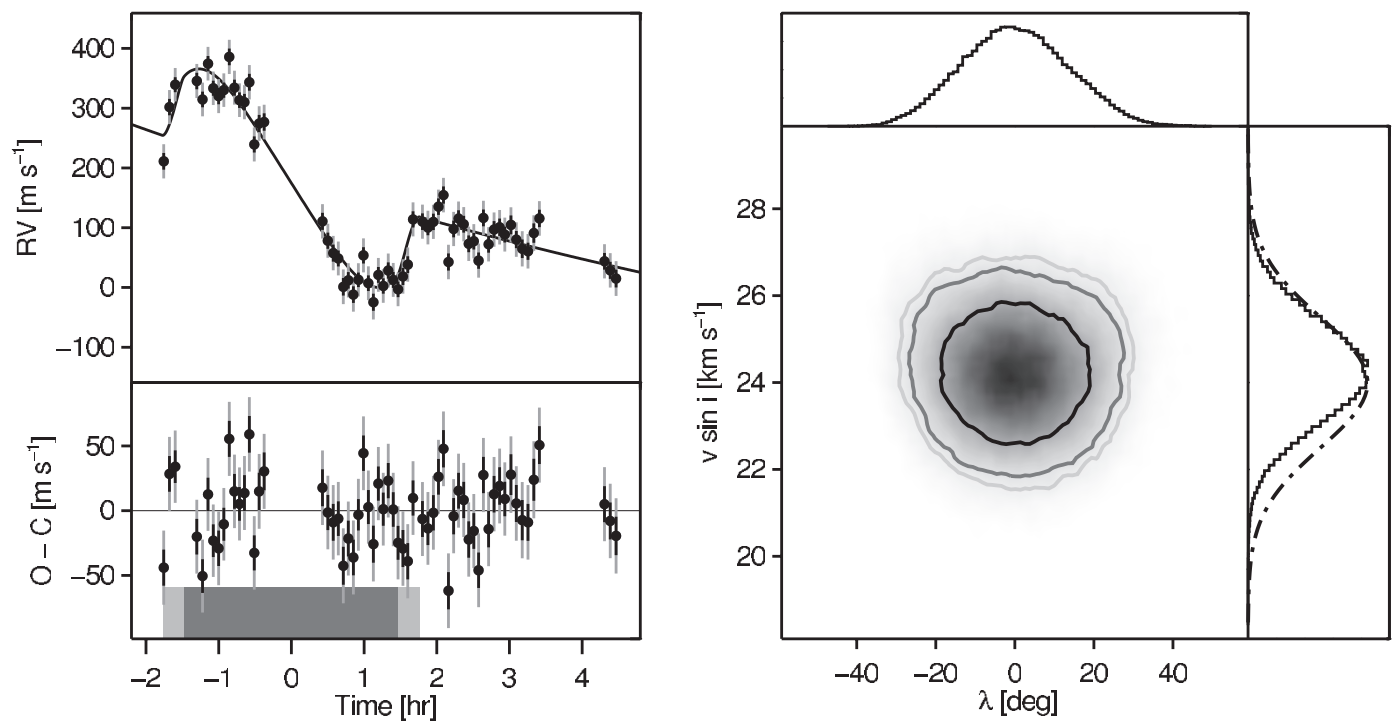

Figure 8. Spectroscopy of HAT-P-34 transit. Similar to Figure 3 but this time for data obtained during a transit in the HAT-P-34 system.

range of angles near $90^{\circ}$ can produce an asymmetric RM effect. Therefore when an asymmetry is detected in such a system, even relatively coarse RV data will lead to high precision in $\lambda$. Interestingly, if we take the $v \sin i_{\star}$ prior at face value and assume that our RM model contains all the relevant physics, then we find with $T_{21}=0.41251 \pm 0.0017 \mathrm{hr}$ a fivefold improvement in our knowledge of the ingress duration compared to the prior constraint $\left(T_{21}=0.4128 \pm 0.0096 \mathrm{hr}\right)$. This is because for a given $v \sin i_{\star}$ and $\lambda$ near $\pm 90^{\circ}$, the impact parameter is encoded in the amplitude of the RM effect.

The pairing of HAT-P-32 and HAT-P-2 is instructive (see Section 4.15). For HAT-P-2 the RM effect was observed with a very high $\mathrm{S} / \mathrm{N}$ : the ratio between the amplitude of the RM effect and the typical RV uncertainty in the RVs is $\sim 10$. But despite the very high $\mathrm{S} / \mathrm{N}$ in the detection of the $\mathrm{RM}$ effect, we obtained a relatively low precision in the measurement of $\lambda$. This is the reverse of what we see for HAT-P-32. For this system, the ratio between the amplitude of the RM effect and the average RV uncertainty is $\sim 2$. Nevertheless we obtain a very high precision in the measurement of $\lambda$. This highlights how important the geometry of the transit together with the projected obliquity is in determining the precision in the measurement of the projected obliquity.

\subsection{HAT-P-34}

HAT-P-34 b was announced by Bakos et al. (2012) together with three other transiting planets. HAT-P-34 b orbits its host star on a highly eccentric $(e=0.44)$ orbit with a relatively long period of 5.5 days. We observed this system with HIRES during the night 2011 September $2 / 3$. We obtained 62 spectra during and after the transit (Figure 8). As the RM curve suggests we find that the projections of the stellar rotation and orbital angular momenta are well aligned $\left(\lambda=0^{\circ} \pm 14^{\circ}\right)$.

\subsection{WASP-12}

WASP-12 harbors a transiting exoplanet with a short orbital period of 1 d. 1 . It was discovered by Hebb et al. (2009). We took from those authors our prior on $v \sin i_{\star}$, while we obtained from 

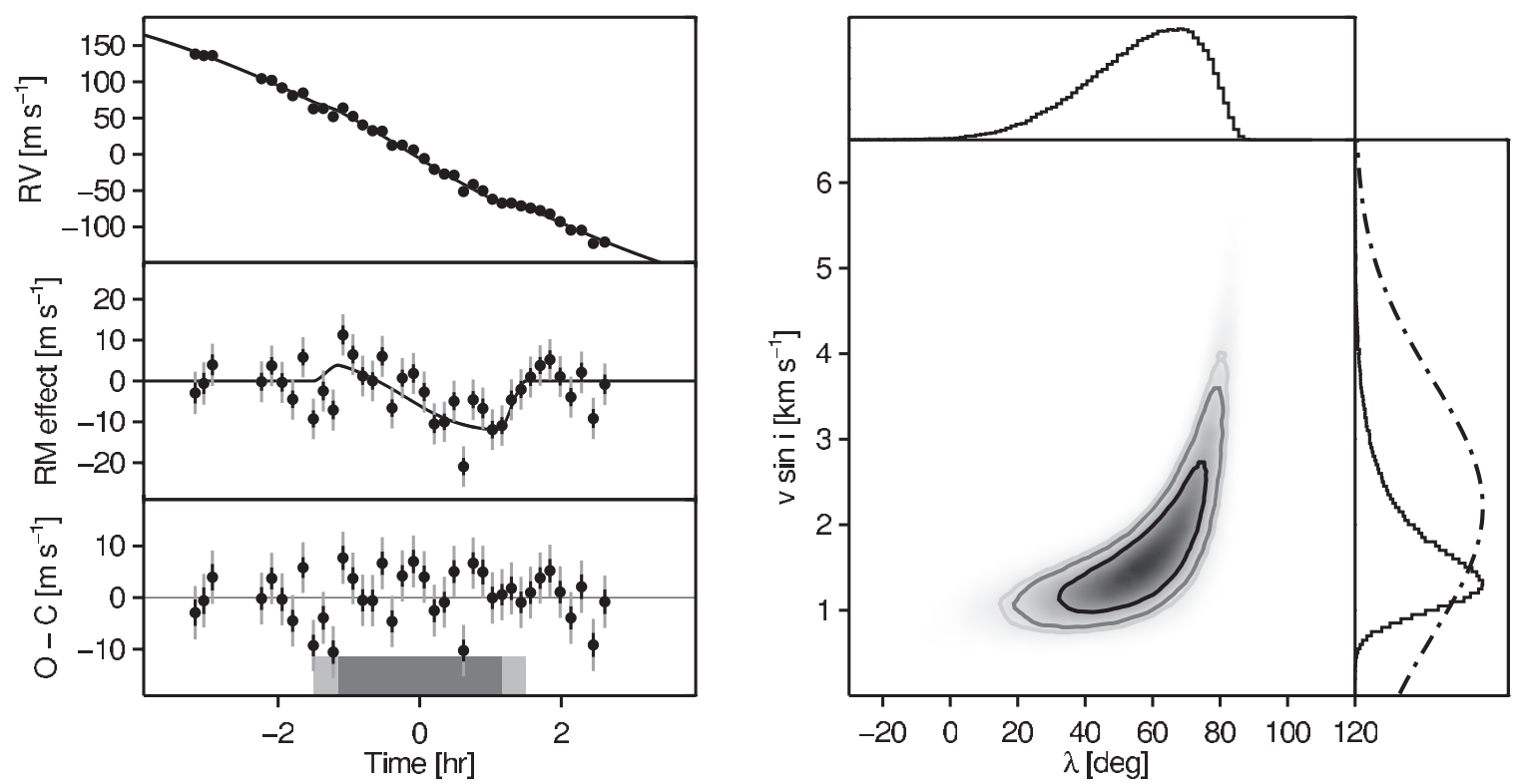

Figure 9. Spectroscopy of WASP-12 transit. Similar to Figure 4 but this time for data obtained during a transit in the WASP 12 system.
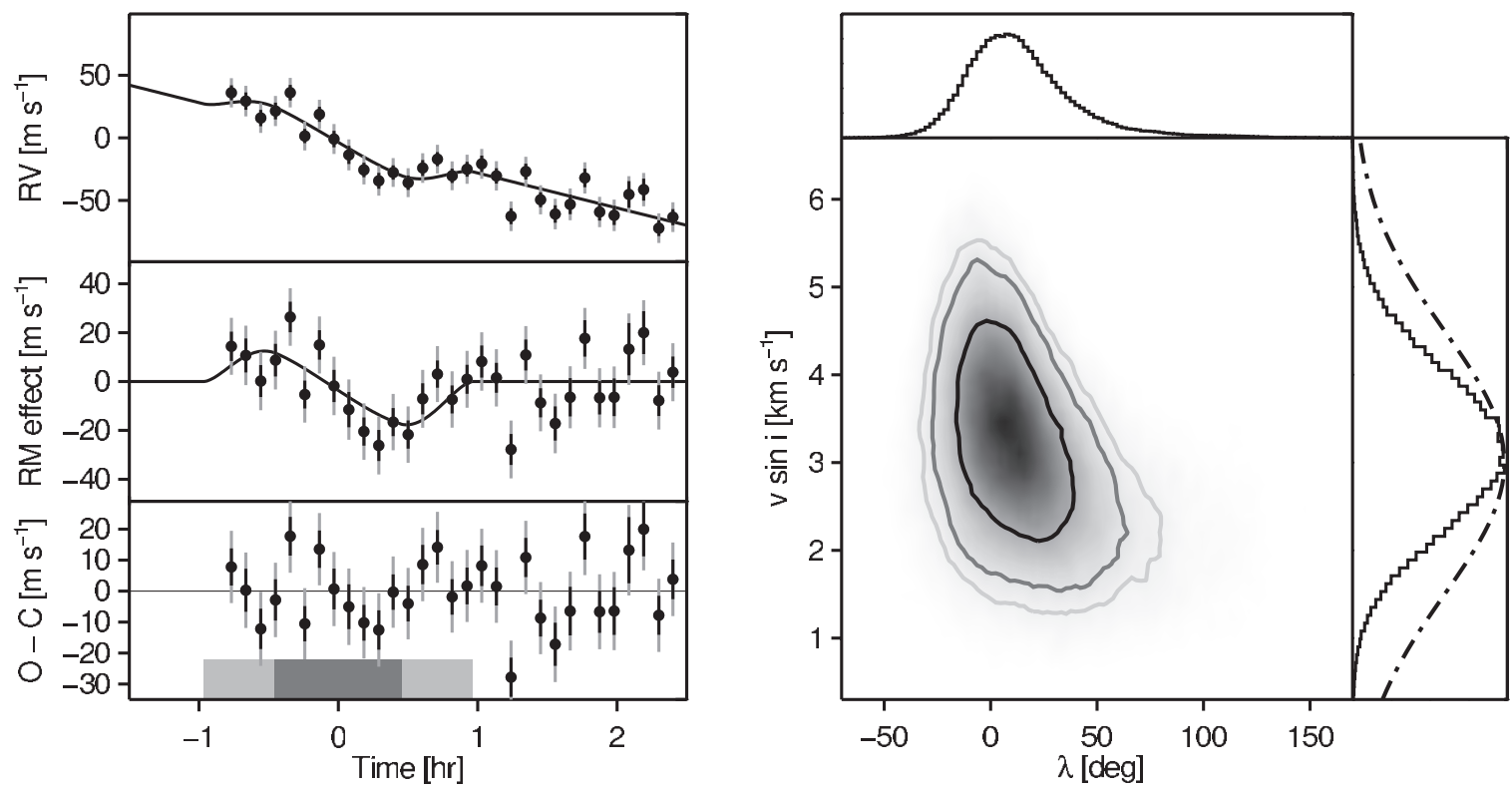

Figure 10. Spectroscopy of WASP-16 transit. Similar to Figure 4 but this time for data obtained during a transit in the WASP 16 system.

Maciejewski et al. (2011) our priors on the geometric transit parameters. ${ }^{12}$

We observed the RM effect in this system on 2012 January $1 / 2$ with the Keck I telescope. Our analysis of the $38 \mathrm{RVs}$ (Figure 9, left panel) obtained before, during, and after the 3 $\mathrm{hr}$ transit indicates a misalignment of $59_{-20}^{+15}$ and $v \sin i_{\star}=$ $1.6_{-0.4}^{+0.8} \mathrm{~km} \mathrm{~s}^{-1}$. For this system there is a strong correlation between $\lambda$ and $v \sin i_{\star}$; for higher projected rotation speeds $\lambda$ increases and approaches $90^{\circ}$ (Figure 9, right panel). This is interesting as WASP-12, with a mass of $1.35 M_{\odot}$ (Maciejewski et al. 2011), is expected to be a fast rotator and to have a high $v$. Using the method employed by Schlaufman (2010) we find an expected $v$ of $13.7 \pm 2.5 \mathrm{~km} \mathrm{~s}^{-1}$ suggesting a low $\sin i_{\star}$, i.e., the stellar spin axis is inclined along the line of sight. It seems very likely that the star and the planetary orbit are misaligned, though

12 Husnoo et al. (2011) also obtained RV data during a transit of this system, but their results were not conclusive. we cannot tell with certainty how much of the misalignment is in the plane of the sky as opposed to the perpendicular direction. A similar situation was found for WASP-1 (Albrecht et al. 2011b).

\subsection{WASP-16}

Lister et al. (2009) found this transiting hot Jupiter on a 3.1 day orbit around a southern-sky star. We used the PFS in conjunction with the Magellan II telescope to obtain RVs during a transit occurring 2010 June $3 / 4$ (Figure 10). We used the ephemeris and information on the projected rotation speed from the discovery paper as prior information in the fit. As no information on $T_{21}$ was given, we used the EULERCAM light curve presented in the discovery paper to establish priors for $T_{41}, T_{21}$, and $R_{\mathrm{p}} / R_{\star}$. This system is one of those for which we find (based on the fit to the out-of-transit observations) an orbital velocity semi-amplitude $\left(K_{1}=353 \pm 54 \mathrm{~m} \mathrm{~s}^{-1}\right)$ that is significantly different from the orbital solution presented in the discovery 

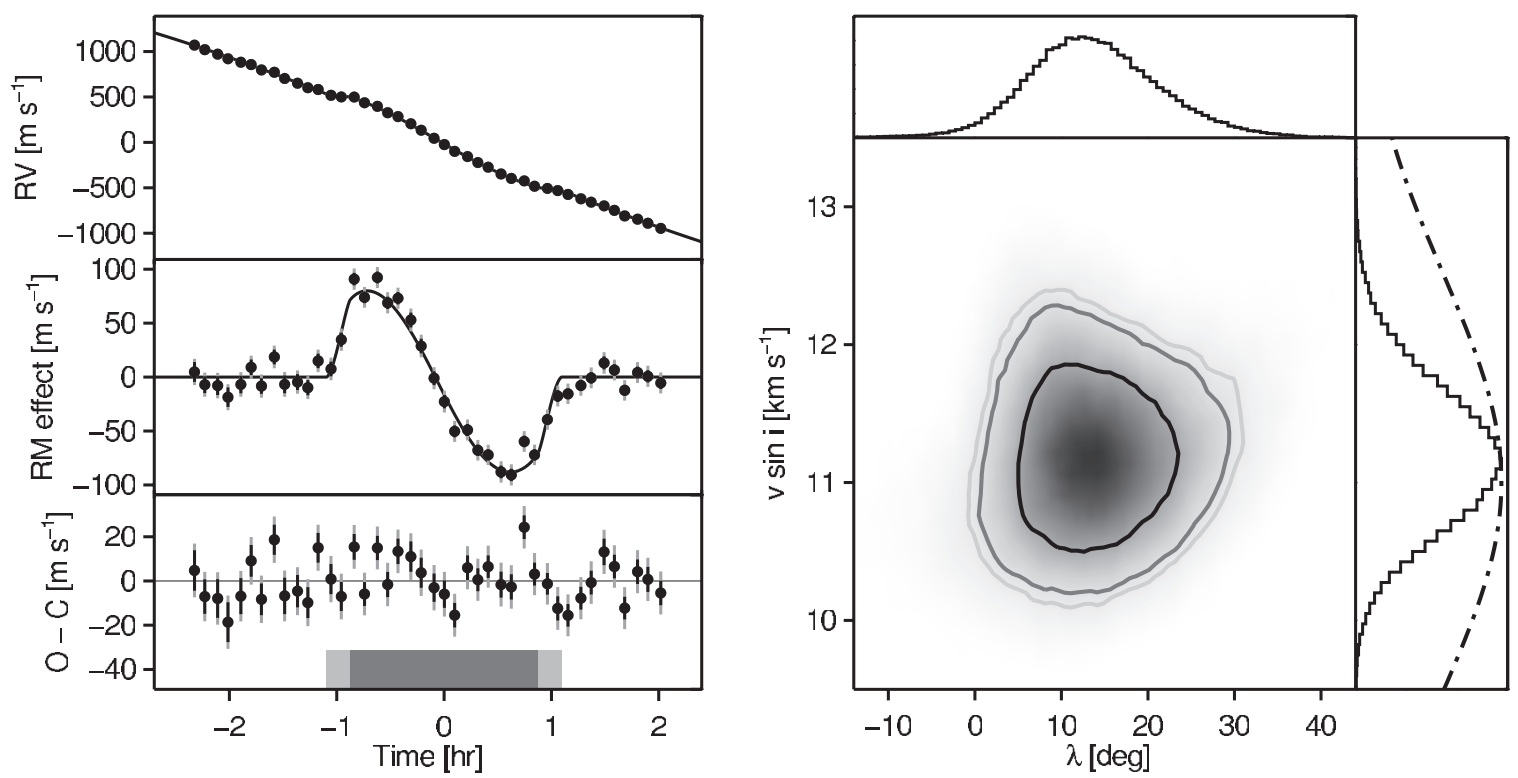

Figure 11. Spectroscopy of WASP-18 transit. Similar to Figure 4 but this time for data obtained during a transit in the WASP 18 system.

paper $\left(K_{1}=116.7_{-1.9}^{+2.4} \mathrm{~m} \mathrm{~s}^{-1}\right)$. To investigate this issue we obtained 10 out-of-transit observations on a number of different nights. These observations agree with the previously reported orbital solution. A large stellar spot might be responsible for the excess RV change during the transit night (see Section 3.5), although such a spot would need to cover a substantial part of the stellar surface.

We omitted nine data points obtained at the end of the transit night, after the template observation for WASP-16 was obtained. These observations would in principle facilitate a separation of the RM signal from other sources of RV variation. However, given that there is already an indication of intrinsic $\mathrm{RV}$ variations apart from orbital motion, and the absence of any guarantee that the intrinsic variations are linear in time over the course of many hours, we decided to use only the data points immediately following egress. All the RVs are listed in Table 2. Using the 31 remaining $\mathrm{RV}$ data points obtained during and after the transit we obtained $\lambda=11_{-19}^{+26}$ and $v \sin i_{\star}=3.2 \pm 0.9 \mathrm{~km} \mathrm{~s}^{-1}$. Brown et al. (2012) recently measured $\lambda=-4.2_{-13.9}^{+11}$ consistent with the value obtained here. However, they found a significantly lower value for $v \sin i_{\star}\left(1.2_{-0.5}^{+0.4} \mathrm{~km} \mathrm{~s}^{-1}\right)$ which is not consistent with their spectroscopic prior nor with ours. We also note that Brown et al. (2012) did not report any disagreement between the out-of-transit velocity gradient observed on the transit night, and the published orbital solution.

\subsection{WASP-18}

WASP-18 b orbits its host star in only 0.94 days (Hellier et al. 2009). As noted by the discoverers it is an excellent system to study the tidal interaction between a gas giant and its host star. Triaud et al. (2010) found the projections of the stellar spin and orbital axes to be aligned $\left(\lambda=4^{\circ} \pm 5^{\circ}\right)$.

We obtained 48 data points with the PFS during the night 2011 October 8/9. Six of these data points were obtained at a significant distance in orbital phase from the transit, and are not included in the fit, for reasons discussed in the preceding section and Section 3.5. Figure 11 presents the remaining 42 RVs obtained before, during, and after the transit.

No value for $T_{21}$ has been reported in the literature for WASP-18 b. We obtained the photometry from Southworth et al. (2009) and performed our own analysis. For the reasons mentioned by Southworth et al. (2010) we did not use this light curve to improve on the ephemeris for this system. The photometric priors reported in Table 3 for this system are derived from the fit to that light curve.

We found that the projected stellar $\operatorname{spin}\left(\lambda=13^{\circ} \pm 7^{\circ}\right)$ appears to be misaligned with the orbital rotation axis, although the statistical significance is marginal. The value for $v \sin i_{\star} 11.0 \pm$ $0.5 \mathrm{~km} \mathrm{~s}^{-1}$ does not give any strong indication of an inclination along the line of sight. Any obliquity in WASP-18 seems to be small.

\subsection{WASP-19}

WASP-19 is another system with a very short period ( 0.78 days) hot Jupiter discovered by the WASP consortium (Hebb et al. 2010). We observed this system with PFS during the transit night 2010 May 20/21 (Figure 12). Our photometric priors were based on results by Hellier et al. (2011). These authors derived the projected obliquity in the same fit that was used to determine the photometric parameters. This could in principle make our observation of $\lambda$ dependent on their obliquity observation. However, given the quality of their photometric and RV data, we expect that the RVs obtained by them have little influence on their results for $T_{41}, T_{21}$, and $R_{\mathrm{p}} / R_{\star}$. We therefore expect our result $\left(\lambda=15^{\circ} \pm 11^{\circ}\right)$ to be independent of their result $(\lambda=4.6 \pm 5.2)$. Our prior on the projected rotation speed was taken from the discovery paper.

As can be seen in Figure 12, there is correlated excess noise in the RVs during pre-egress. This together with the low-S/N detection raises the concern that we might not have detected an RM effect at all, and we are fitting our RM model to noise. We carried out an experiment similar to the one described by Albrecht et al. (2011b) for the WASP-2 system. Briefly, we create 200,000 fake data sets with similar noise characteristics as the real data set, but without an underlying RM effect. We then fitted an RM model to each data set. Form the resulting density distribution we conclude that our RM detection represents a $3 \sigma$ detection. We also used the Bayesian information criterion (BIC) to assess whether the model including the RM effect is preferred over a model without the RM effect (e.g., Brown et al. 2012). The 

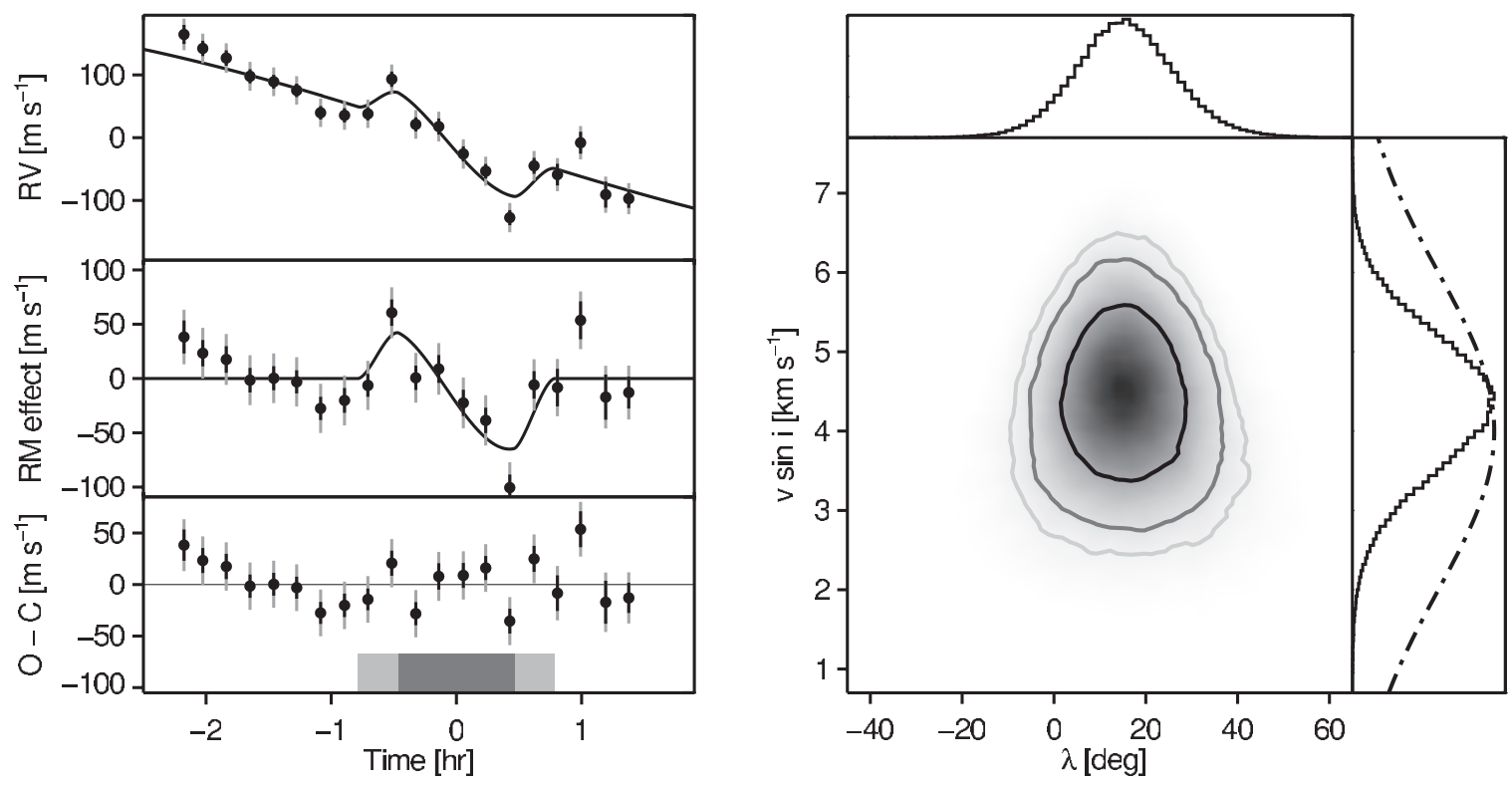

Figure 12. Spectroscopy of WASP-19 transit. Similar to Figure 4 but this time for data obtained during a transit in the WASP 19 system.
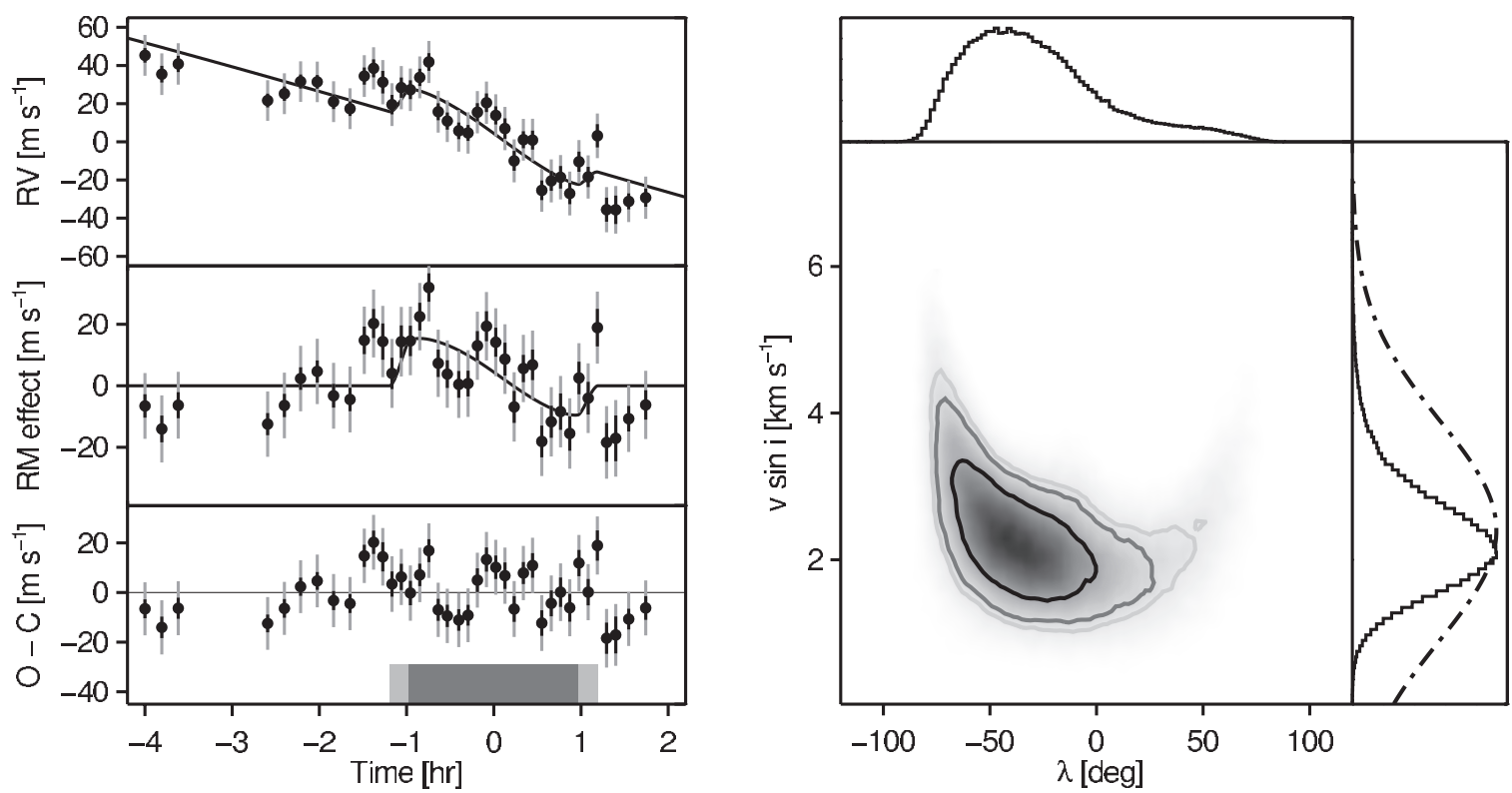

Figure 13. Spectroscopy of WASP-26 transit. Similar to Figure 4 but this time for data obtained during a transit in the WASP 26 system.

$\mathrm{RM}$ model is preferred, with $\mathrm{BIC}_{\mathrm{RM}}=48$ and $\mathrm{BIC}_{\mathrm{no} \text { RM }}=60$. However, the BIC test depends somewhat on how many of the RV measurements were obtained during transit, compared to the number obtained outside of transit. Furthermore, both tests (or at least our implementations of these tests) assume that the noise is uncorrelated, which does not seem to be the case for the present data set.

\subsection{WASP-26}

This system was detected by Smalley et al. (2010). Anderson et al. (2011a) attempted a measurement of the RM effect but did not make a secure detection. We obtained $39 \mathrm{RV}$ data points during the night 2010 August 17/18 (Figure 13). Using these RVs, photometric priors from Anderson et al. (2011a), and a prior on the projected stellar rotation speed from Smalley et al. (2010) $)^{13}$ we derive $\lambda=-34_{-26}^{+36}$ and $v \sin i_{\star}=2.2 \pm 0.7 \mathrm{~km} \mathrm{~s}^{-1}$.

\subsection{WASP-31}

The existence of WASP-31 b was announced by Anderson et al. (2011b). We observed this system with the Keck I telescope during the night 2012 March 12/13 and obtained 27 RV measurements (Figure 14).

Using these RVs together with the $v \sin i_{\star}, T_{41}, T_{21}$, and $R_{\mathrm{p}} / R_{\star}$ priors from Anderson et al. (2011b) and priors on the ephemeris

13 We used the $v \sin i_{\star}$ value from Smalley et al. (2010) as prior, and not the value reported by Anderson et al. (2011a). This is because the former authors estimated $v \sin i_{\star}$ under the assumption that the macroturbulent parameter is $4.1 \pm 0.3 \mathrm{~km} \mathrm{~s}^{-1}$. This is closer to our estimation $\left(5.1 \pm 1.5 \mathrm{~km} \mathrm{~s}^{-1}\right)$ than the value of $3.0 \pm 0.3 \mathrm{~km} \mathrm{~s}^{-1}$ used by the latter authors. 

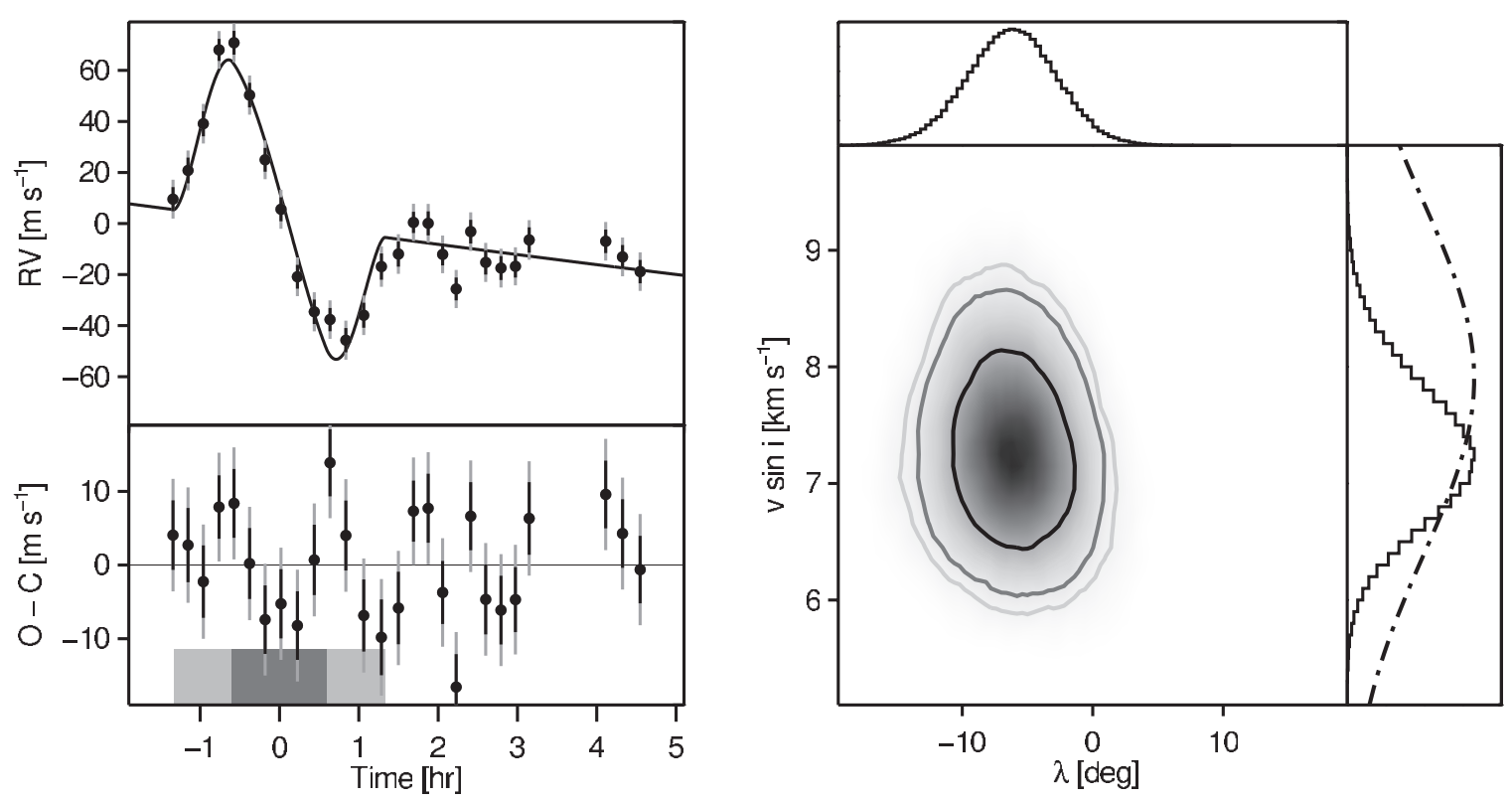

Figure 14. Spectroscopy of WASP-31 transit. Similar to Figure 3 but this time for data obtained during a transit in the WASP 31 system.
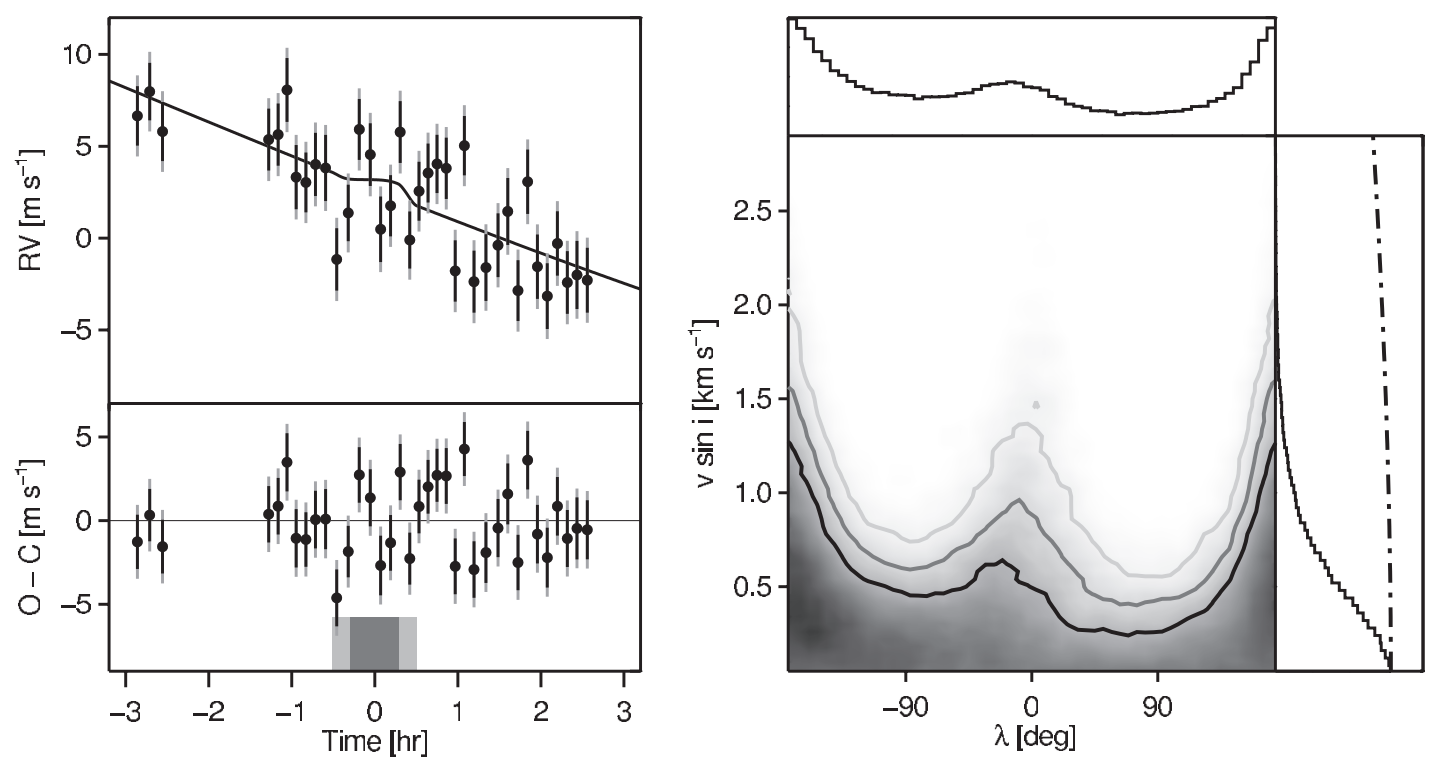

Figure 15. Spectroscopy of Gl 436 transit. Similar to Figure 3 but this time for data obtained during a transit in the GI 436 system.

from Dragomir et al. (2011) we found a low projected obliquity $\left(\lambda=-6^{\circ} \pm 3^{\circ}\right)$. We obtained $v \sin i_{\star}=7.3 \pm 0.4 \mathrm{~km} \mathrm{~s}^{-1}$, consistent with the prior $\left(7.9 \pm 1.5 \mathrm{~km} \mathrm{~s}^{-1}\right)$. Brown et al. (2012) recently found $\lambda=2.8 \pm 3.1$ and $v \sin i_{\star}=7.5 \pm 0.7 \mathrm{~km} \mathrm{~s}^{-1}$. The value for $\lambda$ is inconsistent with our result. Brown et al. (2012) used the ephemeris presented in Anderson et al. (2011b). Using the $T_{c}$ and $P$ values from Anderson et al. (2011b) to set the constraint on the time of inferior conjunction for the observed spectroscopic transit we obtain $\lambda=2^{\circ} \pm 3^{\circ}$. This is consistent with the result by Brown et al. (2012). For our final results we decided to use the timing information from Dragomir et al. (2011), who used the ephemeris from the discovery paper in combination with an additional light curve (although we note that the new light curve contains only a small amount of postegress data).

Therefore while we have excellent agreement between two different research groups, the dependence of the result on the photometric priors and the inconsistency between the results using the same RVs but different timing information cast some doubt on the formal uncertainty intervals. For this reason, we double the uncertainty in $\lambda$ before including this system in the subsequent discussion of the interpretation of all the results. Future photometric observations will be helpful, but at least it seems clear that the projected obliquity is small.

\subsection{3. $G l 436$}

The transiting planet in Gl 436 was discovered by Butler et al. (2004) and found to be transiting by Gillon et al. (2007). It would be of interest to know the obliquity in this system as Gl $436 \mathrm{~b}$ is of similar mass then HAT-P-11 b, which is so far the only Neptune-class planet for which the host star's obliquity has been measured. Using HIRES we obtained RVs of the system during the night of 2010 April 24/25. To reduce the uncertainties in the photometric parameters, we gathered new photometric data with Keplercam, a CCD camera on the $1.2 \mathrm{~m}$ telescope of 

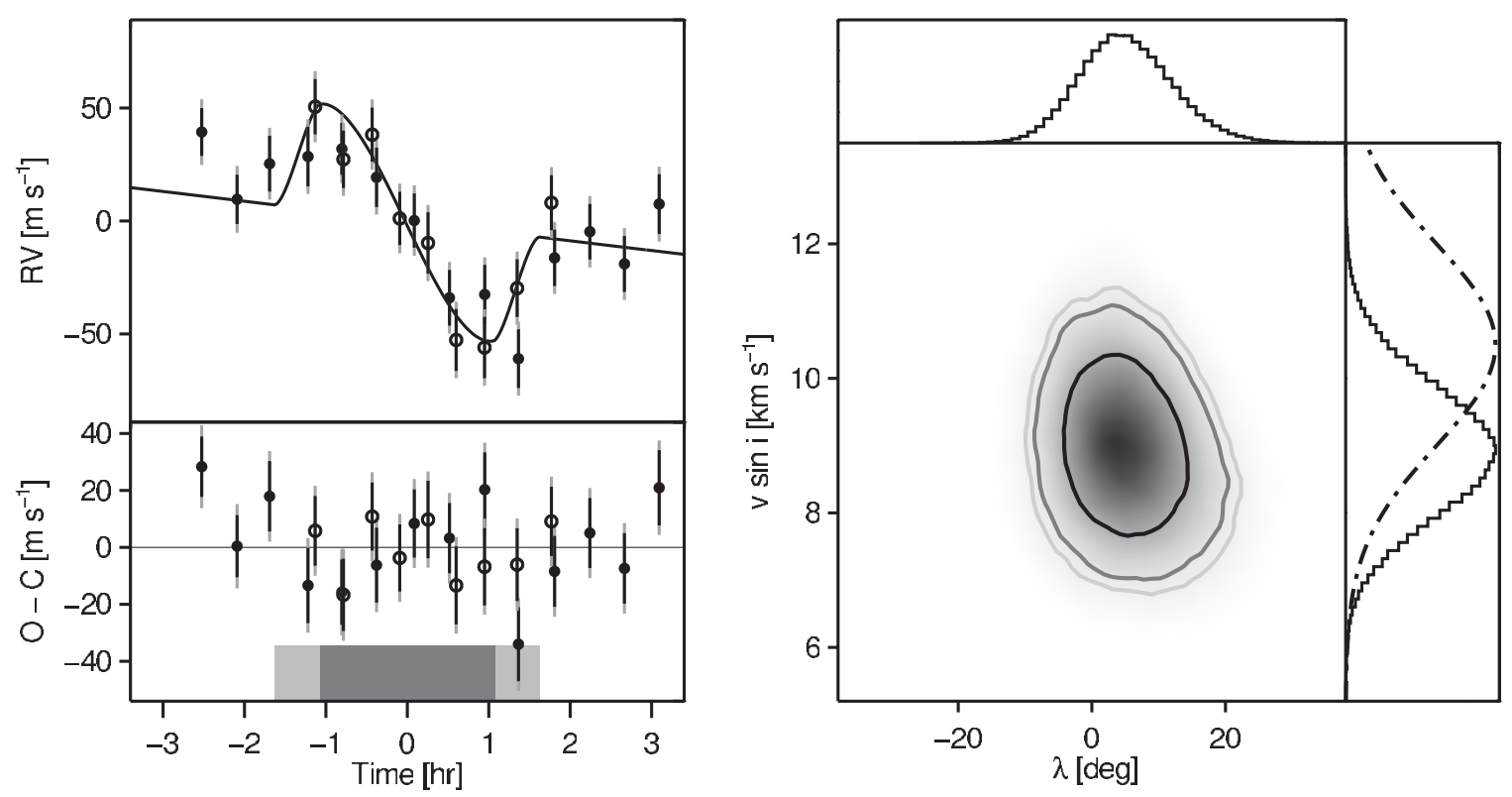

Figure 16. Spectroscopy of Kepler-8 transit. Similar to Figure 3 but this time for data obtained during a transit in the Kepler-8 system. Black filled circles represent the RV data points already obtained by Jenkins et al. (2010), while the open circles show the new HIRES data.

the Fred L. Whipple Observatory on Mount Hopkins, Arizona (Szentgyorgyi et al. 2005). The RV measurements are displayed in the left panels of Figure 15. The right panels display the posterior in the $\lambda-v \sin i_{\star}$ plane. No RM effect was detected, and therefore the data provide no constraint on $\lambda$. Only an upper limit of $0.4 \mathrm{~km} \mathrm{~s}^{-1}$ on $v \sin i_{\star}$ can be obtained. This upper limit on $v \sin i_{\star}$ is more constraining than the prior we adopted, which was a one-sided Gaussian prior enforcing $v \sin i_{\star}<5 \mathrm{~km} \mathrm{~s}^{-1}$. Thus from the transit data we learned only that the star is a slow rotator. The structure in the posterior distribution is caused by the lower sensitivity of our measurement for nearly prograde and retrograde configurations. See Albrecht et al. (2011b) for an explanation of this effect.

\subsection{Kepler-8}

Jenkins et al. (2010) reported the discovery of Kepler- 8 and also found $\lambda=-26^{\circ} \pm 10.1$, a misalignment between the projections of the stellar and orbital spins. As the data set available to Jenkins et al. (2010) only consisted of data taken during the transit itself, it is difficult to assign a significance to the result. We therefore obtained a new data set with HIRES during the transit night 2011 August 7/8. We used both HIRES data sets in our analysis, including two additional parameters representing a second velocity offset and a second RV transitnight velocity gradient between the two different transit nights. Flat priors were assigned to these parameters. We found that the data are consistent with good alignment between the stellar and orbital rotation axes (see Figure 16). We obtained $\lambda=5^{\circ} \pm 7^{\circ}$ and $v \sin i_{\star}=8.9 \pm 1.0 \mathrm{~km} \mathrm{~s}^{-1}$. The velocity offset between the two data sets was found to be $12 \pm 10 \mathrm{~m} \mathrm{~s}^{-1}$. Using only the new data set in our analysis, we obtained $\lambda=5^{\circ} \pm 10^{\circ}$. Using only the older data set, we obtained $\lambda=30_{-28}^{+55^{\circ}}$. We chose to use both data sets for this system, as they have been obtained with the same instrument and setup, and the RVs were extracted with the same code, circumstances different from all the other systems for which multiple transits have been observed. Furthermore this case highlights the importance of prior constraints on the data. Without a constraint on the RV offset as applied by Jenkins et al. (2010), the original data set is consistent with good alignment.

\subsection{HAT-P-2}

HAT-P-2 was one of the first systems for which a measurement of the projected obliquity was reported. Winn et al. (2007) measured $\lambda=1.2 \pm 13^{\circ} .4$. Loeillet et al. (2008) found $\lambda=0.2_{-12.5}^{+12.2 \circ}$, a similar value. The uncertainty in this measurement is relatively high because HAT-P-2 $b$ has a low-impact parameter. After these results were published, new and improved system parameters based on new photometry were reported by Pál et al. (2010). For this reason we reanalyzed the RVs obtained by Winn et al. (2007) together with the new photometric priors, obtaining $\lambda=9^{\circ} \pm 5^{\circ}$. Thanks to the improved photometric information, the formal uncertainty in $\lambda$ is now lower, and supports a $1.8 \sigma$ "detection" of a misalignment, a different conclusion from the one by Winn et al. (2007). However, one can see in the lower left panel of Figure 17 that, after subtraction of our best fitting model, structure remains in the residuals.

This is a clear sign that our model does not capture all the effects influencing the RV anomaly during transit. We therefore consider the uncertainty of our analysis to be itself quite uncertain, by at least a factor of two. In Table 4 we report a doubled uncertainty relative to our formal results for $\lambda$ and $v \sin i_{\star}$. Therefore while the photometric information as well as the quality of the RV data would allow for a measurement of $\lambda$ to within a few degrees, the limitations of our model of the RM effect prevent this gain from being fully realized. The model expects a larger RM effect shortly before and after mid-transit and a lower amplitude around the second and third contacts. One possible effect that would produce this type of structured residuals is a change of limb darkening with depths of stellar absorption lines. We note that no such structure in the residuals was seen in the original analysis of these data using a different modeling for the RM effect, which was derived empirically for the specific spectrograph and stellar type (Winn et al. 2007).

It is not surprising that the limitations of our model would be most apparent for HAT-P-2, as it has the combination of very rapid rotation and very high $\mathrm{S} / \mathrm{N}$, given the brightness of the star $(V=8.7)$. For this system it would be better to analyze the absorption line profiles directly, and their changes over the 

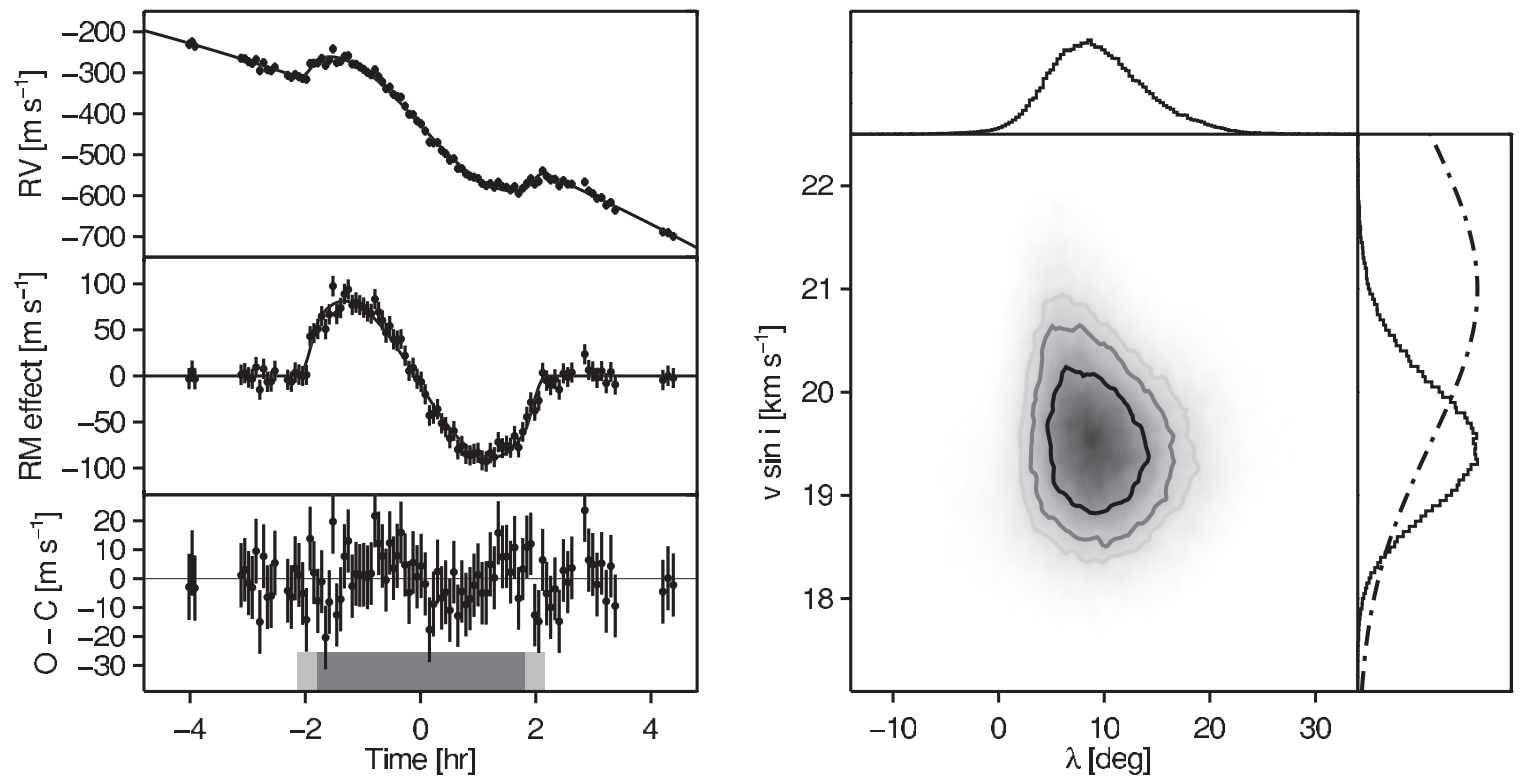

Figure 17. Spectroscopy of HAT-P-2 transit. Similar to Figure 4 but this time for data obtained during a transit in the HAT-P-2 system. Structure can be seen in the residuals indicating that our modeling does not capture all the relevant physics of the RM effect.
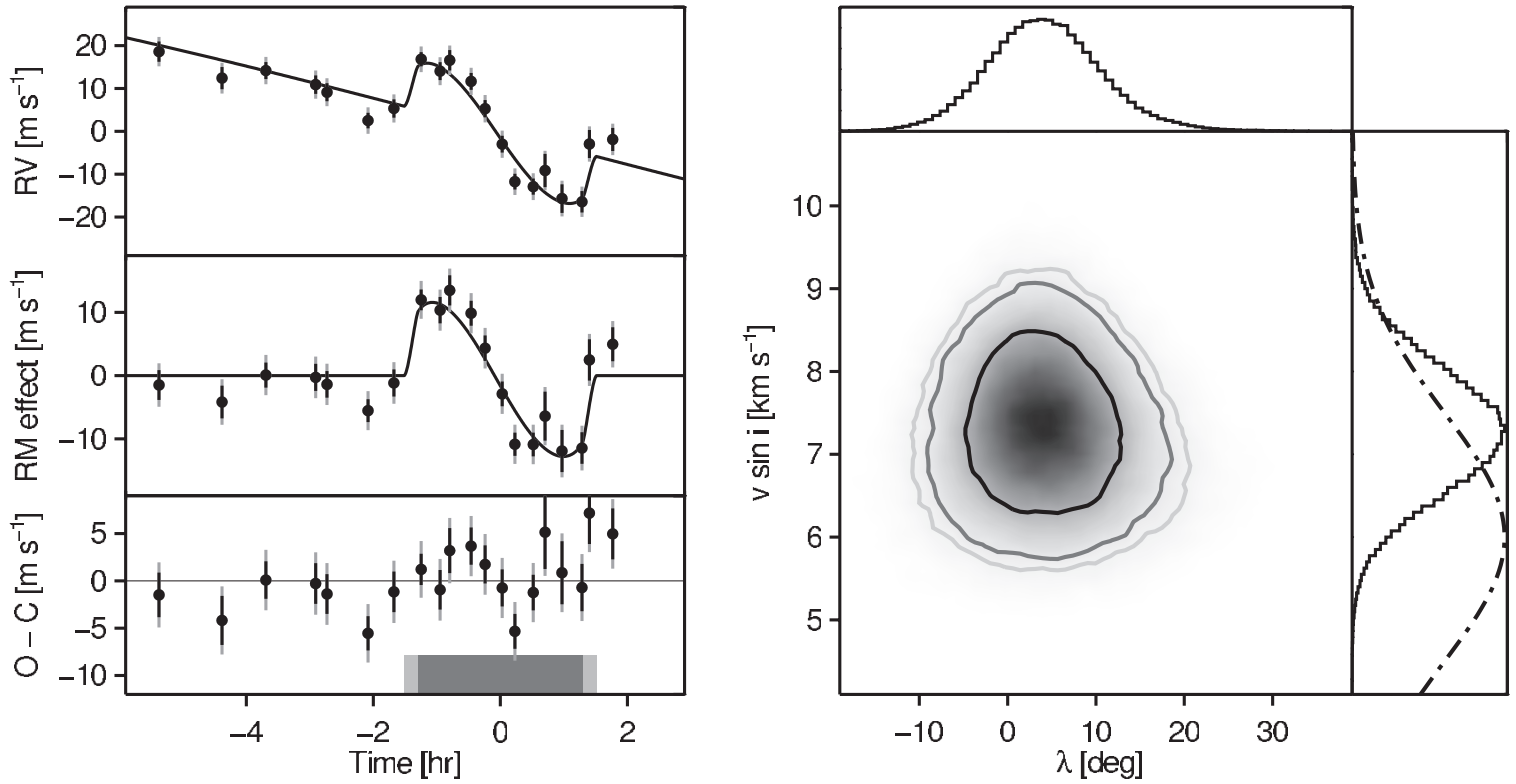

Figure 18. Spectroscopy of HD 149026 transit. Similar to Figure 4 but this time for data obtained during a transit in the HD 149026 system.

course of the night, rather than deriving and modeling RVs (e.g., Albrecht et al. 2007; Collier Cameron et al. 2010).

\subsection{6. $H D 149026$}

Wolf et al. (2007) found $\lambda=-12^{\circ} \pm 15^{\circ}$ for this well-known system. Since that time, (Carter et al. 2009) presented a more precise light curve based on Hubble Space Telescope (HST) infrared observations. Thus a re-analysis is warranted. We used the photometric information from Carter et al. (2009) and the $v \sin i_{\star}$ prior from the discovery paper (Sato et al. 2005). We found a projected obliquity of $12^{\circ} \pm 7^{\circ}$. This is only marginally consistent with the previously reported value of $\lambda$ using the same $\mathrm{RV}$ data. The main reasons for the difference are the use of new photometric data, and the lack of post-egress data (Figure 18) in combination with our choice to not impose a prior constraint on $K_{\star}$. When we repeated the analysis with a prior on $K_{\star}$ (Table 4) we found the results to be consistent with those of Wolf et al. (2007).

\subsection{HD 209458}

The first system in which a transiting planet was discovered was also the first system for which the projected obliquity was measured. Queloz et al. (2000) found an angle consistent with alignment to within $20^{\circ}$. Winn et al. (2005) found $\lambda$ to be $-4.4 \pm 1.4$. The later result suggested that a small but significant misalignment exists in this system, similar to the misalignment of our Sun against the ecliptic $\left(\sim 7^{\circ}\right)$. However, Winn et al. (2005) used data from different nights, unlike our current procedure. If the star is active or has star spots, the longterm intrinsic RV noise would be manifested as velocity offsets between different nights, which were not taken into account by Winn et al. (2005). Indeed we find evidence that the stellar jitter has different amplitudes on different timescales: by fitting 

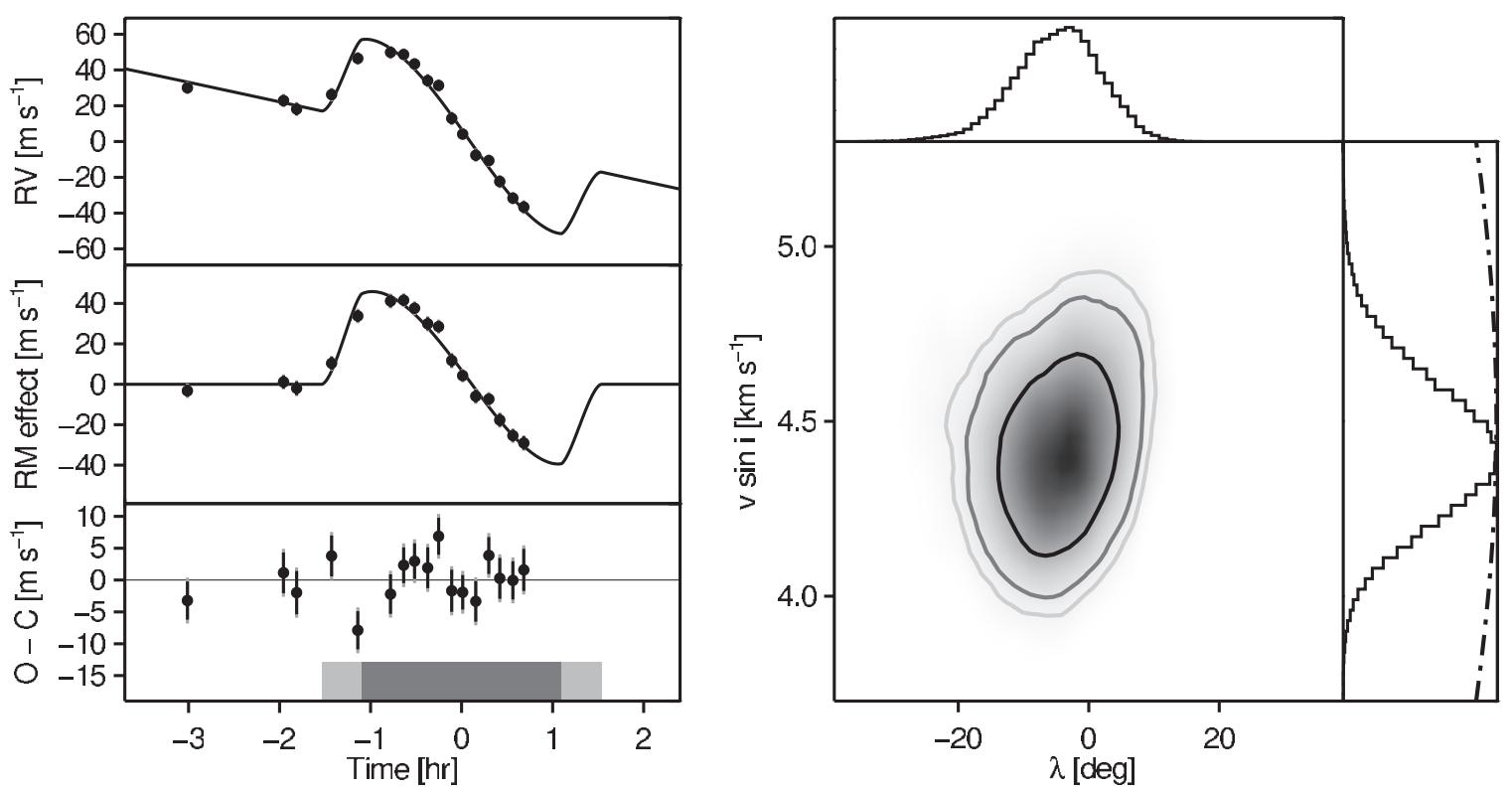

Figure 19. Spectroscopy of HD 209458 transit. Similar to Figure 4 but this time for data obtained during a transit in the HD 209458 system.

a model to all of the available HIRES data, we find the rms residual of the data obtained on different nights to be $5 \mathrm{~m} \mathrm{~s}^{-1}$ while the rms residual of the transit-night data is only $3 \mathrm{~m} \mathrm{~s}^{-1}$.

We therefore repeated the analysis using only the HIRES RVs obtained during the transit night, 2000 July 29/30. We also used the HST light curve obtained by Brown et al. (2001) to obtain photometric priors, and used the $v \sin i_{\star}$ value from Laughlin et al. (2005) as a prior. We obtained a projected stellar obliquity of $-5^{\circ} \pm 7^{\circ}$. The looser bounds on $\lambda$ are a consequence of the lack of egress and post-egress data on the night 2000 July 29/30 (Figure 19). This case emphasizes the need to obtain data outside of the transit, unless one is willing to assume that the long-term $\mathrm{RV}$ noise is negligible.

\section{DISCUSSION}

We now use the measurements of projected obliquities obtained in the last section together with other measurements taken from the literature to learn more about the evolution of these systems. ${ }^{14}$

In Section 5.1, we first remark on some specific cases from the literature. In Section 5.2, we revisit the evidence for a correlation between the degree of alignment and the effective stellar temperature discovered by Winn et al. (2010a). We then discuss further evidence of tidal interaction, based on correlations between the planet-to-star mass ratio and $\lambda$, and the dependence of $\lambda$ on the orbital distance between the two bodies. In Section 5.2, we sort the systems according to a theoretical tidal timescale, as a test of whether tides have been important in altering the obliquities of these systems. We also discuss the implications for the origin of hot Jupiters and the strengths and weaknesses of our interpretation.

\subsection{Remarks on the Previous Literature}

CoRoT-1. The RM effect was measured by Pont et al. (2010). We do not include this measurement in our subsequent

\footnotetext{
14 We present here all measurements with the sign convection form Ohta et al (2005) and use the symbol $\lambda$. Often researchers use the symbol $\beta$ and the sign convention from Hosokawa (1953), where $\lambda=-\beta$.
}

discussion because of the low $\mathrm{S} / \mathrm{N}$ of the detection and because it was only possible to obtain a few out-of-transit observations. The authors caution that systematic uncertainties could cause the actual errors in the measurement of the projected obliquity $\left(\lambda=77^{\circ} \pm 11^{\circ}\right)$ to be larger than the statistical uncertainty. We note that if the value for $\lambda$ from this study were taken at face value, it would constitute an exception to the pattern presented below. For this reason it would be an important system to reobserve. It is a challenging target because of the faint and slowly rotating host star.

CoRoT-11. Gandolfi et al. (2010) obtained RVs during a planetary transit in this system. As only the first half of the transit was observed, and only with a low $\mathrm{S} / \mathrm{N}$, they could only conclude that the orbit was prograde. In that sense this is a similar case to HAT-P-16 presented here. We decided to exclude CoRoT-11 from subsequent discussion as we did with our result on HAT-P-16.

CoRoT-19. This system has an F9V star and a Jupitermass planet on a 3.9 day orbit. The RM effect was measured by Guenther et al. (2012). They found $\lambda=-52_{-22}^{+27}{ }^{\circ}$, a misalignment between stellar and orbital axes. However, the RM effect was only detected at the $2.3 \sigma$ level, and no postegress data were obtained. We omit this measurement in the subsequent discussion.

KOI-13. Szabó et al. (2011) detected a slight asymmetry in the transit light curve and attributed the asymmetry to a misalignment of the planet's orbit relative to the stellar rotation. The host star is a fast rotator, leading to a lower surface gravity and surface brightness around the equator compared to the poles. By modeling this effect, Barnes et al. (2011) found $|\lambda|$ to be either $24^{\circ} \pm 4^{\circ}$ or $156^{\circ} \pm 4^{\circ}$. Either choice represents a substantial misalignment and would lead to similar conclusions in the subsequent discussion. For simplicity of presentation in the plots to follow, we arbitrarily adopt the lower value. Barnes et al. (2011) also calculated the stellar inclination along the line of sight, which we do not use here, since this information is not available for most of the other systems. We adopt the mass for the secondary estimated by Mislis \& Hodgkin (2012), which is also consistent with work by Shporer et al. (2011). Both estimates were based on the photometric orbit. We 
further adopt the age estimate of $\left(710_{-150}^{+180} \mathrm{Myr}\right)$ by Szabó et al. (2011).

WASP-23. We omit the measurement by (Triaud et al. 2011). Because of a low-impact parameter, the only conclusion that could be drawn is that orbit is prograde.

$X O-2$. We omit the measurement by (Narita et al. 2011). They found $\lambda=10^{\circ} \pm 72^{\circ}$, a prograde orbit but with a very large uncertainty, similar to our result for HAT-P-16.

\subsection{Relevant System Properties}

Effective temperature. Winn et al. (2010a) noted that for hot Jupiters, nonzero values of $\lambda$ tended to be associated with hot stars. For effective temperatures $\gtrsim 6250 \mathrm{~K}$ the obliquities have a broad distribution, while for lower temperatures the measurements are consistent with low obliquities. The only exceptions were two systems with significantly longer orbital periods than the rest. Schlaufman (2010) independently found that hot stars tended to be misaligned with the orbits of hot Jupiters by comparing the measured value of $v \sin i_{\star}$ with the expected value for $v$, for a star of the given mass and age. While this approach has the virtue of requiring less intensive observations, it does rely heavily on accurate measurement of $v \sin i_{\star}$, which is problematic for slowly rotating stars, and assumes that $v$ was not affected by any tidal influence of the close-in planet.

With the new measurements presented in this paper and with measurements by others over the last two years, the number of systems with measured projected obliquities is now up to 53. This is more than twice the number that was available to Winn et al. (2010a). Table 5 shows the measured projected obliquities of all systems used in this study. ${ }^{15}$ The increased number of measurements enables a stringent test of the proposed pattern, as well as a more in depth analysis and comparison to other system parameters.

It should be noted that in almost all cases, the measurements are of the projected obliquity, and not the true obliquity. For true obliquities smaller than $90^{\circ}$ the projected obliquity is usually smaller than the true obliquity, while for true obliquities $>90^{\circ}$ the projected obliquity is usually larger than the true obliquity. This factor complicates any detailed comparison between the measurements and the theoretical expectations. For simplicity we have chosen to work with projected obliquities, rather than attempting any deprojection scheme (Fabrycky \& Winn 2009; Morton \& Johnson 2011).

The upper panel of Figure 20 shows the projected obliquities plotted as a function of the effective temperature of the host star. Apparently, for these systems, the trend observed by Winn et al. (2010a) still holds. There are three apparent exceptions to the rule: HAT-P-11, WASP-8, and HD 80606. These systems are special in other ways too, by virtue of having either an unusually low planet mass or an unusually long orbital period. They represent three of the four systems for which the orbital period is greater than 7 days or the planet has a mass lower than $0.2 M_{\text {Jup }}$. In this sense they least resemble the typical "hot Jupiter." We will discuss these important cases in the following paragraphs.

The explanation for the relationship between $T_{\text {eff }}$ and $\lambda$ could fall into one of two categories: (1) the formation and evolution of hot Jupiters is different for hot stars than for cool stars, which for some reason results in higher obliquities in the hot stars.

\footnotetext{
15 See also exoplanets.org, exoplanet.eu, www.astro.keele.ac.uk/jkt/tepcat,
} and www.aip.de/People/rheller for listing of obliquity measurements.
Table 5

Measured Projected Obliquities

\begin{tabular}{|c|c|c|c|c|}
\hline & System & $\begin{array}{c}v \sin i_{\star} \\
\left(\mathrm{km} \mathrm{s}^{-1}\right)\end{array}$ & $\begin{array}{c}\lambda \\
\left(^{\circ}\right)\end{array}$ & Reference \\
\hline 1 & CoRoT-2 b & $10.9 \pm 0.5$ & $4.0_{-6.1}^{+5.9}$ & 1 \\
\hline 2 & CoRoT-3 b & $17.0 \pm 1.0$ & $37.6_{-22.3}^{+10}$ & 2,3 \\
\hline 3 & CoRoT-18 b & $8.0 \pm 1.0$ & $-10 \pm 20$ & 4 \\
\hline 4 & HAT-P-1 b & $3.8 \pm 0.6$ & $3.7 \pm 2.1$ & 5 \\
\hline 5 & HAT-P-2 b & $19.5 \pm 1.4$ & $9 \pm 10$ & This work \\
\hline 6 & HAT-P-4 b & $5.8 \pm 0.3$ & $-4.9 \pm 11.9$ & 6 \\
\hline 7 & HAT-P-6 b & $7.8 \pm 0.6$ & $165 \pm 6$ & This work \\
\hline 8 & HAT-P-7 b & $2.7 \pm 0.5$ & $155 \pm 37$ & This work \\
\hline 9 & HAT-P-8 b & $14.5 \pm 5$ & $-17_{-11.5}^{+9.2}$ & 7 \\
\hline 10 & HAT-P-9 b & $12.5 \pm 1.8$ & $-16 \pm 8$ & 7 \\
\hline 11 & HAT-P-11 b & $1.0 \pm 0.9$ & $103_{-10}^{+26}$ & 8 \\
\hline 12 & HAT-P-13 b & $1.7 \pm 0.4$ & $1.9 \pm 8.6$ & 9 \\
\hline 13 & HAT-P-14 b & $8.2 \pm 0.5$ & $-170.9 \pm 5.1$ & 6 \\
\hline 14 & HAT-P-16 b & $3.9 \pm 0.8$ & $-10 \pm 16$ & 7 \\
\hline 15 & HAT-P-23 b & $7.8 \pm 1.6$ & $15 \pm 22$ & 7 \\
\hline 16 & HAT-P-24 b & $11.2 \pm 0.9$ & $20 \pm 16$ & This work \\
\hline 17 & HAT-P-30 b & $3.1 \pm 0.2$ & $73.5 \pm 9.0$ & 10 \\
\hline 18 & HAT-P-32 b & $20.6 \pm 1.5$ & $85 \pm 1.5$ & This work \\
\hline 19 & HAT-P-34 b & $24.3 \pm 1.2$ & $0 \pm 14$ & This work \\
\hline 20 & HD $17156 b$ & $4.1 \pm 0.3$ & $10 \pm 5.1$ & 11 \\
\hline 21 & HD 80606 b & $1.7 \pm 0.3$ & $42 \pm 8$ & 12 \\
\hline 22 & HD 149026 b & $7.7 \pm 0.8$ & $12 \pm 7$ & This work \\
\hline 23 & HD 189733 b & $3.1 \pm 0.1$ & $-0.5 \pm 0.4$ & 13 \\
\hline 24 & HD $209458 \mathrm{~b}$ & $4.4 \pm 0.2$ & $-5 \pm 7$ & This work \\
\hline 25 & KOI-13 b & $65 \pm 10$ & $24 / 154 \pm 4$ & 14 \\
\hline 26 & Kepler-8 b & $8.9 \pm 1.0$ & $5 \pm 7$ & This work \\
\hline 27 & Kepler-17 b & $4.7 \pm 1.0$ & $<15$ & 15 \\
\hline 28 & TrES-1 b & $1.1 \pm 0.3$ & $30 \pm 21$ & 16 \\
\hline 29 & TrES-2 b & $2 \pm 1$ & $-9 \pm 12$ & 17 \\
\hline 30 & TrES-4 b & $8.5 \pm 1.2$ & $6.3 \pm 4.7$ & 18 \\
\hline 31 & WASP-1 b & $0.7 \pm 1.4$ & $-59 \pm 99$ & -26 \\
\hline 32 & WASP-3 b & $14.1 \pm 1.5$ & $3.3_{-4.4}^{+2.5}$ & 20 \\
\hline 33 & WASP-4 b & $2.1 \pm 0.4$ & $-1_{-12}^{+14}$ & 21,22 \\
\hline 34 & WASP-5 b & $3.2 \pm 0.3$ & $12.1_{-10}^{+8}$ & 22 \\
\hline 35 & WASP-6 b & $1.6 \pm 0.3$ & $-11_{-14}^{+18}$ & 23 \\
\hline 36 & WASP-7 b & $14 \pm 2$ & $86 \pm 8$ & 24 \\
\hline 37 & WASP-8 b & $20 \pm 0.6$ & $-123_{-4.4}^{+3.4}$ & 25 \\
\hline 38 & WASP-12 b & $1.6_{-0.4}^{+0.8}$ & $59_{-20}^{+15}$ & This work \\
\hline 39 & WASP-14 b & $2.8 \pm 0.6$ & $-33.1 \pm 7.4$ & 26 \\
\hline 40 & WASP-15 b & $4.3 \pm 0.4$ & $-139.6_{-5.2}^{+4.3}$ & 22 \\
\hline 41 & WASP-16 b & $3.2 \pm 0.9$ & $11_{-19}^{+26}$ & This work \\
\hline 42 & WASP-17 b & $9.9 \pm 0.5$ & $-148.7_{-6.7}^{+7.7}$ & 27 \\
\hline 43 & WASP-18 b & $11.2 \pm 0.6$ & $13 \pm 7$ & This work \\
\hline 44 & WASP-19 b & $4.4 \pm 0.9$ & $15 \pm 11$ & This work \\
\hline 45 & WASP-22 b & $4.4 \pm 0.3$ & $22 \pm 16$ & 28 \\
\hline 46 & WASP-24 b & $7 \pm 0.9$ & $-4.7 \pm 4$ & 29 \\
\hline 47 & WASP-25 b & $7 \pm 0.9$ & $14.6 \pm 6.7$ & 30 \\
\hline 48 & WASP-26 b & $2.2 \pm 0.7$ & $-34_{-26}^{+36}$ & This work \\
\hline 49 & WASP-31 b & $6.8 \pm 0.6$ & $-6 \pm 6$ & This work \\
\hline 50 & WASP-33 b & $86 \pm 1$ & $-107.7 \pm 1.6$ & 31 \\
\hline 51 & WASP-38 b & $8.6 \pm 0.4$ & $15_{-43}^{+33}$ & 29 \\
\hline 52 & $\mathrm{XO}-3 \mathrm{~b}$ & $17.0 \pm 1.2$ & $37.3 \pm 3.0$ & 32 \\
\hline 53 & $\mathrm{XO}-4 \mathrm{~b}$ & $8.8 \pm 0.5$ & $-46.7 \pm 8.1$ & 33 \\
\hline
\end{tabular}

Reference. (1) Gillon et al. 2010; (2) Triaud et al. 2009; (3) Deleuil et al. 2008; (4) Hébrard et al. 2011b; (5) Johnson et al. 2008; (6) Winn et al. 2011b; (7) Moutou et al. 2011; (8) Winn et al. 2010c; (9) Winn et al. 2010b; (10) Johnson et al. 2011; (11) Narita et al. 2009a; (12) Hébrard et al. 2010; (13) Collier Cameron et al. 2010; (14) Barnes et al. 2011; (15) Désert et al. 2011; (16) Narita et al. 2007; (17) Winn et al. 2008; (18) Narita et al. 2010; (19) Albrecht et al. 2011b; (20) Tripathi et al. 2010; (21) Sanchis-Ojeda et al. 2011; (22) Triaud et al. 2010; (23) Gillon et al. 2009; (24) Albrecht et al. 2012; (25) Queloz et al. 2010; (26) Johnson et al. 2009; (27) Anderson et al. 2011c; (28) Anderson et al. 2011a; (29) Simpson et al. 2011; (30) Brown et al. 2012; (31) Collier Cameron et al. 2010; (32) Hirano et al. 2011a; (33) Narita et al. 2010. 


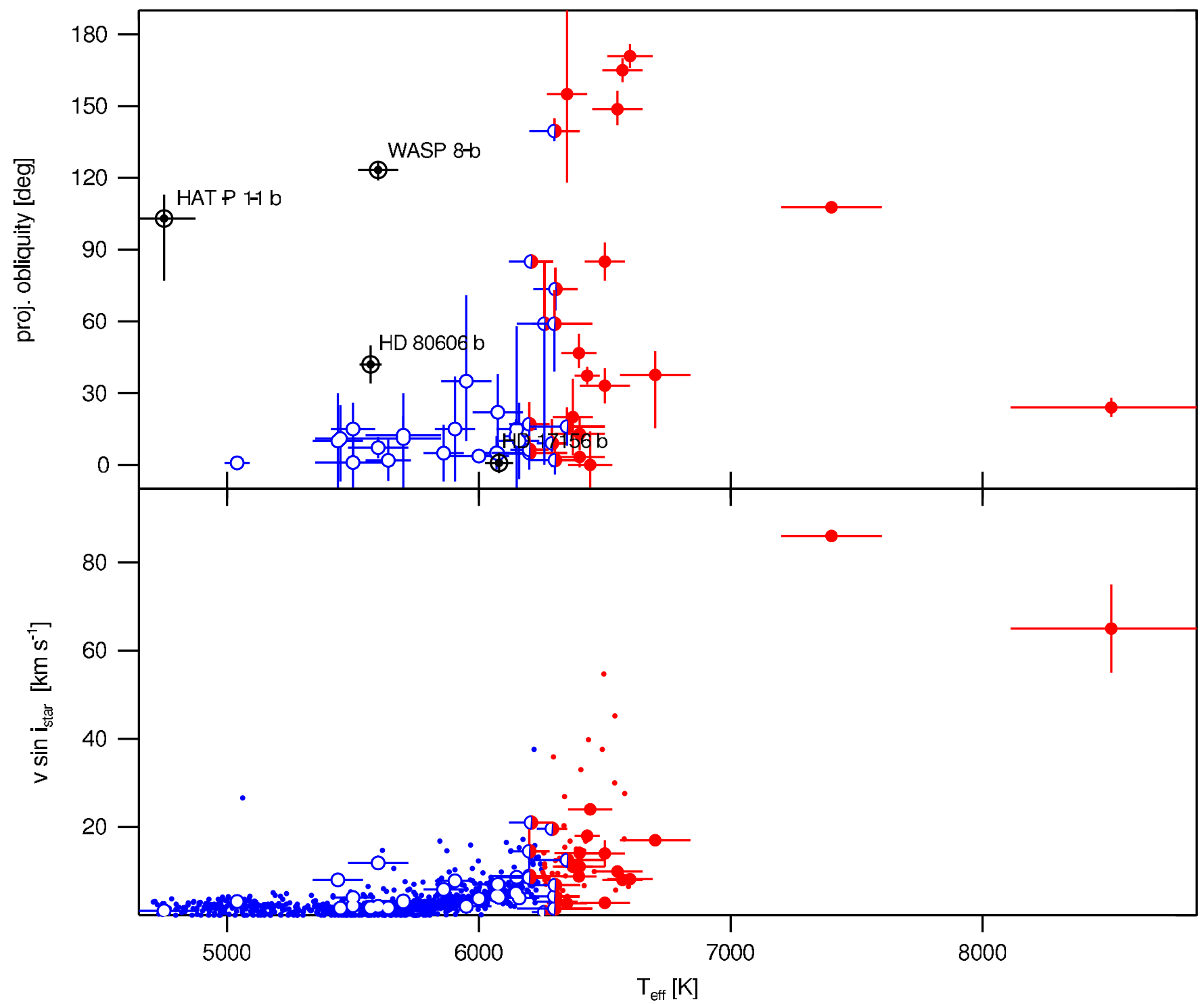

Figure 20. Projected obliquities and projected stellar rotation speeds as a function of the stellar effective temperature. Upper panel: measurements of projected obliquities as a function of the effective temperature of the host star. Stars which have temperatures higher then $6250 \mathrm{~K}$ are shown with red filled symbols. Blue open symbols show stars with temperatures lower then $6250 \mathrm{~K}$. Stars which measured effective temperature include $6250 \mathrm{~K}$ in their $1 \sigma$ interval are shown by split symbols. Systems which harbor planets with mass $<0.2 M_{\text {Jup }}$ or have an orbital period more then 7 days are marked by a black filled circle with a ring. Lower panel: projected stellar rotation speeds $v \sin i_{\star}$ of the stars in our sample. In addition, $v \sin i_{\star}$ measurements of stars in the catalog by Valenti \& Fischer (2005) are shown as small dots. (A color version of this figure is available in the online journal.)

(2) The distribution of obliquities is originally broad for both hot stars and cool stars, but they evolve differently depending on a parameter closely associated with $T_{\text {eff }}$. Winn et al. (2010a) suggested that the second scenario is more likely and that the factor associated with temperature is the rate of tidal dissipation due to the tide raised by the planet.

The reason for this suspicion was that $T_{\text {eff }} \approx 6250 \mathrm{~K}$ is not an arbitrary temperature, but rather represents an approximate boundary over which the internal structure of a main-sequence star changes substantially. Stars hotter than this level have very thin or absent convective envelopes, with the mass of the envelope dropping below about $0.002 M_{\odot}$ at $6250 \mathrm{~K}$ (Pinsonneault et al. 2001). (For the Sun, the mass of the convective envelope is around $\left.0.02 M_{\odot}\right)$.

Independently of this theoretical expectation, there is dramatic empirical evidence for a transition in stellar properties across the $6250 \mathrm{~K}$ divide: hotter stars are observed to rotate more rapidly. In the lower panel of Figure 20, we plot the pro- jected rotation speeds of a sample of $\sim 1000$ stars from the catalogue by Valenti \& Fischer (2005). The projected rotational speed $v \sin i_{\star}$ increases rapidly around $6250 \mathrm{~K}$. For F0 stars, the rotation speed can approach $200 \mathrm{~km} \mathrm{~s}^{-1}$. It is thought that stellar rotation together with the convection in the envelope create a magnetic field coupling to the ionized stellar wind far beyond the stellar radius and thereby transport angular momentum away from the stellar rotation (see, e.g., Barnes 2003, for further discussion.) Presumably this magnetic braking is less effective for stars without convective envelopes, leading to the observed rapid increase in stellar rotation speeds toward earlier spectral type. Judging from Figure 20, the transition from low obliquity to high obliquity seems to be linked empirically to this transition from slowly rotating to rapidly rotating stars.

The presence of a convective envelope is also expected to change the rate of dissipation of the energy in tidal oscillations. Energy contained in tidal bulges is thought to be more effectively dissipated by turbulent eddies in convective envelopes than 


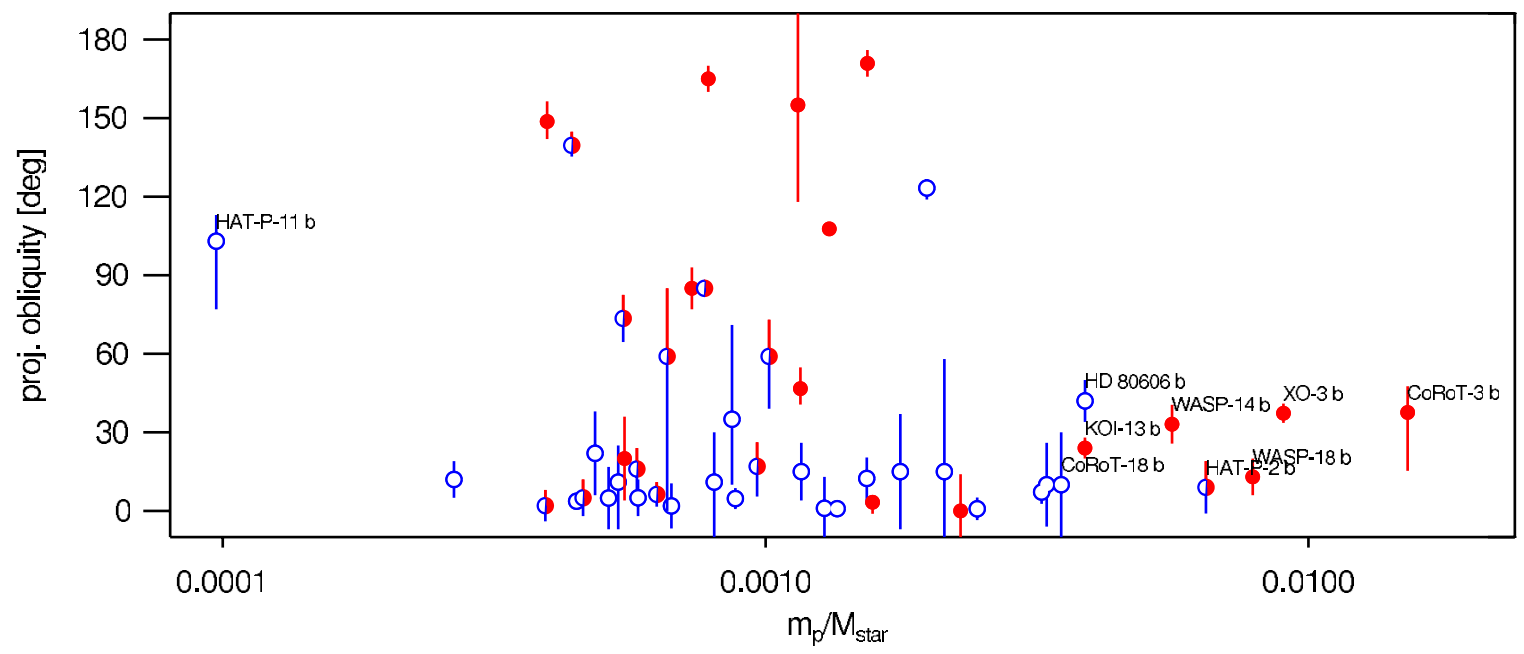

Figure 21. Projected obliquities as function of the planetary to stellar mass ratio. The same symbols as in Figure 20 are used. With larger mass of the companion the degree of misalignment decreases, while for some of the more massive planets a small but significant obliquity is detected. These planets orbit stars with radiative envelopes, reducing the effectiveness with which tidal energy can be dissipated. Names of systems with particular small or large mass ratios are indicated.

(A color version of this figure is available in the online journal.)

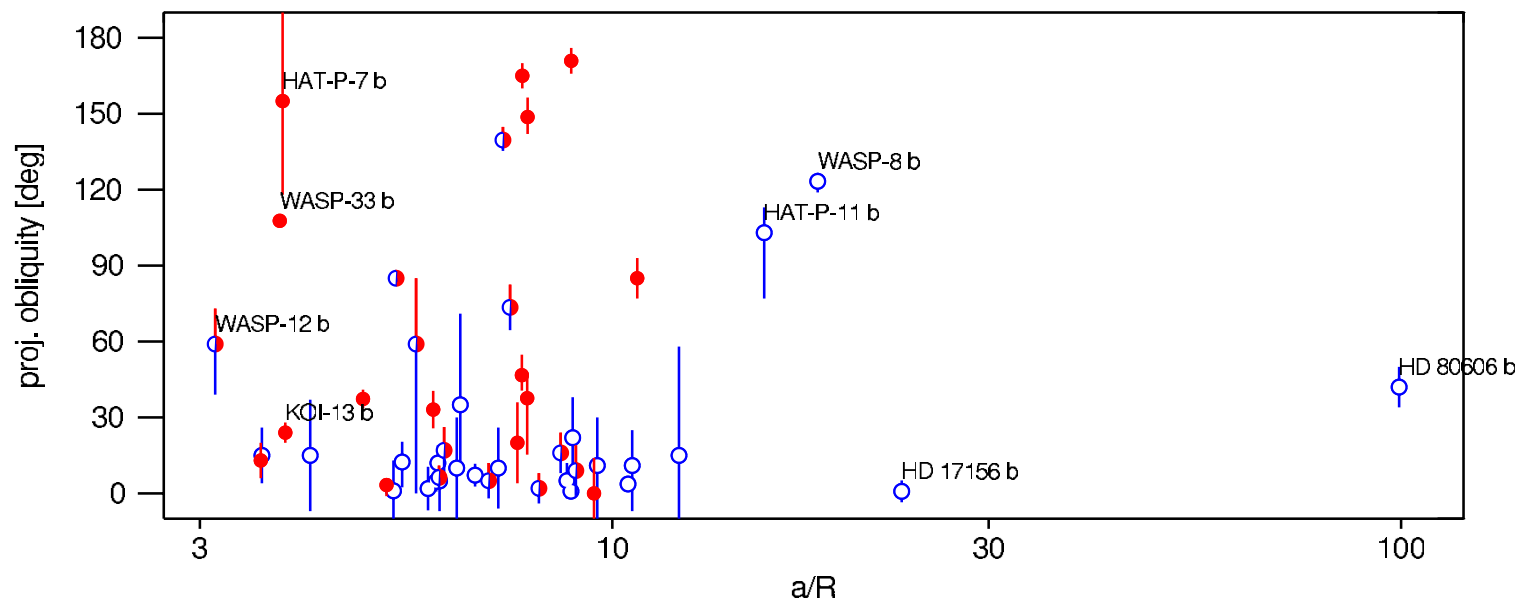

Figure 22. Dependence of $\lambda$ on the scaled orbital distance. The same as Figure 21, but now the measurements of the projected obliquity are plotted as a function of the semi-major axis divided by the stellar radius. While a trend with distance can be observed for planets around cooler stars, such a trend seems to be absent for planets around hotter stars. This might be due to the small range probed in distance by the obliquity measurements around hot stars. However, because tidal forces are weak in these stars only for the innermost massive planets (e.g., WASP-12 b and WASP-18 b) such a trend would be readily observable. Names of systems with scaled distances greater then 15 and for misaligned close-in hot systems are indicated.

(A color version of this figure is available in the online journal.)

by any mechanism acting in a radiative envelope. See Zahn (2008) for a review on the theory of tidal interactions. Torres et al. (2010) and Mazeh (2008) review the evidence for tidal interactions in double-star systems, and provide more access points to the literature.

If tidal evolution is responsible for the difference in stellar obliquities between cool and hot stars, then there should also be a correlation between the mass ratio of the star and planet and the degree of alignment. The higher the mass of the planet $\left(m_{\mathrm{p}}\right)$ relative to the mass of the $\operatorname{star}\left(M_{\star}\right)$, the faster its tides can align the stellar and orbital angular momentum vectors. Furthermore, one would expect an inverse correlation between the scaled semi-major axis $\left(R_{\star} / a\right)$ and the obliquity. A more distant companion will raise smaller tides. We will next investigate whether such correlations exist. We will also investigate if the age of the systems is an important factor in setting the degree of alignment in these systems.

Mass ratio. In Figure 21 the measured projected obliquities are plotted as a function of the mass ratio between planet and host star. Higher obliquities are measured for systems in which the mass of the planet is relatively small. This is what would be expected if tides are responsible for the obliquity distribution. Massive planets raise stronger tides. This trend was observed before (e.g., Hébrard et al. 2011a), though at that time it was not interpreted in terms of tidal interaction.

The interpretation is not clear from this comparison alone, though. Note for example that the most massive planets are found around hot stars, which should have weaker dissipation that counteracts the effect of the more massive planet to at least some degree. In a subsequent section we will attempt to take both these effects (and that of orbital distance) into account at once.

Distance dependence. Does the degree of alignment depend on the scaled distance $\left(a / R_{\star}\right)$ ? Figure 22 gives a mixed answer to this question. Focusing on systems with cool stars (blue open circles) there seems to be a trend of obliquities as a function of the scaled distance. The data suggest good alignment for all systems with $a / R_{\star}<10$. Three of the four systems with greater distances have significant misalignments. 


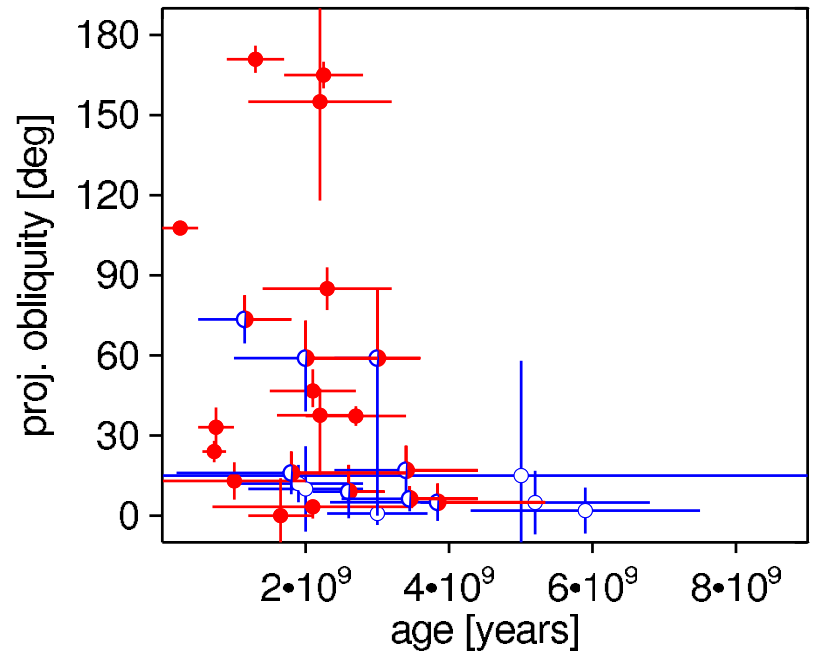

Figure 23. Projected obliquity plotted as function of age for stars with $M_{\star}>1.2 M_{\odot}$. This is a similar plot to the one presented by Triaud (2011). Same symbols used as in Figure 20. Systems which are older than $\sim 3$ Gyr are cool enough to develop a convective envelope. This plot is therefore a relative to Figure 20.

(A color version of this figure is available in the online journal.)

No such dependence is observed for systems with stars which are close to $6250 \mathrm{~K}$ or hotter. However, the range of distances that is spanned by the hot-star systems is very small, less than an order of magnitude. In contrast, the cool-star systems probe nearly two orders of magnitude. Note that two of the closein, misaligned systems are the systems with the hottest host stars (WASP-33 and KOI-13). This lack of alignment finds an explanation in the tidal hypothesis: despite the tight orbits, the tidal dissipation rate may be relatively low due to thin or nonexistent convective layer.

Age. Under the tidal hypothesis, older systems should tend to be closer to alignment than younger systems, all else being equal. This is because in older systems, tides have had a longer interval over which to act. Included in "all else being equal" is the underlying assumption that the arrival time of the hot Jupiter to its close-in orbit is the same in all systems.

Triaud (2011) presented empirical evidence that the degree of misalignment depends chiefly on the age of the system. He found that all systems in his sample with ages greater than $2.5 \mathrm{Gyr}$ are aligned (see his Figure 2). His sample consisted only of those stars with an estimated mass greater than $1.2 M_{\odot}$, since it is harder to determine a reliable age for lower-mass systems.

Stars with a mass of $\gtrsim 1.2 M_{\odot}$ develop a significant convective envelope during their main-sequence lifetime, even if they were too hot to have a significant convective envelope on the "zero age" main sequence. In Figure 23 we plot the projected obliquities as function of stellar age for stars with $M_{\star}>1.2 M_{\odot}$. And indeed all the aligned and older systems are cool enough to have a significant convective envelope. Figure 23 represents, therefore, a similar pattern as seen in Figure 20 with a slight shift in the variable and for a subset of systems (only stars with $M_{\star}>1.2 M_{\odot}$ ). It seems as though the development of a convective envelope with age, rather than the age itself, might be driving the degree of alignment.

\subsection{Tidal Timescale}

As we have seen in the last section that the degree of alignment is correlated with stellar temperature, the mass ratio, and possibly the orbital distance. We now try in this section to establish a single quantitative relationship between the degree of alignment and those parameters. Ideally we could calculate a theoretical alignment timescale for each system, and compare that timescale to the estimated age of the system. We could then check if systems with a relatively short timescale (fast alignment) tend to have low obliquities, and systems with timescales comparable to the lifetime of the system (or larger) tend to have high obliquities.

Calculating timescales needed to synchronize and align stellar rotation is a complex task. Apart from the parameters mentioned above, there are other parameters that would influence the time needed for alignment. For example the total amount of angular momentum stored in the stellar rotation, and the driving frequency of the tidal force (i.e., twice the orbital frequency), are expected to be important. In addition, the rate of dissipation is not expected to be constant over Gyr timescales due to the contemporaneous evolution in orbital distance and eccentricity, and due to stellar evolution. Even worse, the specific mechanisms for dissipating tidal energy are not completely understood, either for stars with radiative envelopes or for stars with convective envelopes. Nevertheless, there are some simple considerations we may employ to obtain approximate timescales for alignment.

1. We can use the formulae provided by Zahn (1977) for synchronization. The coefficients in these formulae are difficult to derive from theory alone, but they can be calibrated with observations made in binary star systems. By observing the maximum orbital distance within which binary stars are observed to be spin-orbit synchronized, and knowing the lifetime of the stars on the main sequence, the relevant parameters can be estimated. To apply this to our sample two different formulae are needed. One for cool stars which have convective envelopes (CE) and hot stars which have radiative envelopes (RA). Therefore, this approach has the virtue of empirical calibration, although the calibration is for star-star interactions rather than planet-star interactions, and the calibration is for spin synchronization rather than reorientation. One complication is that to apply these formulae we have to make a binary decision on whether a star is "convective" or "radiative" which does not reflect the gradual thinning of the convective envelope with increasing stellar temperature. We choose a temperature of $6250 \mathrm{~K}$ for this boundary.

2. Assuming that the alignment timescale due to dissipation in convective envelopes $\left(\tau_{\mathrm{CE}}\right)$ is always shorter than the time needed for alignment by forces in radiative envelopes $\left(\tau_{\mathrm{RA}}\right)$ we can try to derive a simple relationship between the mass contained in the convective envelope and the alignment timescale $\tau$. This would have the advantage that the gradual decrease of mass in the convective envelope can be easily incorporated, but we ignore here any possible additional dissipation mechanism in the radiative envelope which for higher temperatures would become important. In addition it is not obvious why $\tau^{-1}$ should depend linearly on the mass contained in the convective envelope, nor can we be completely confident in our estimate of the convective mass based only on the observable parameters of the stellar photosphere. And of course the convective mass is not really a constant over Gyr timescales.

The simplifications made by either approach should cause us not to expect a perfect and deterministic relationship between our theoretical parameters and the observed obliquities (and we 


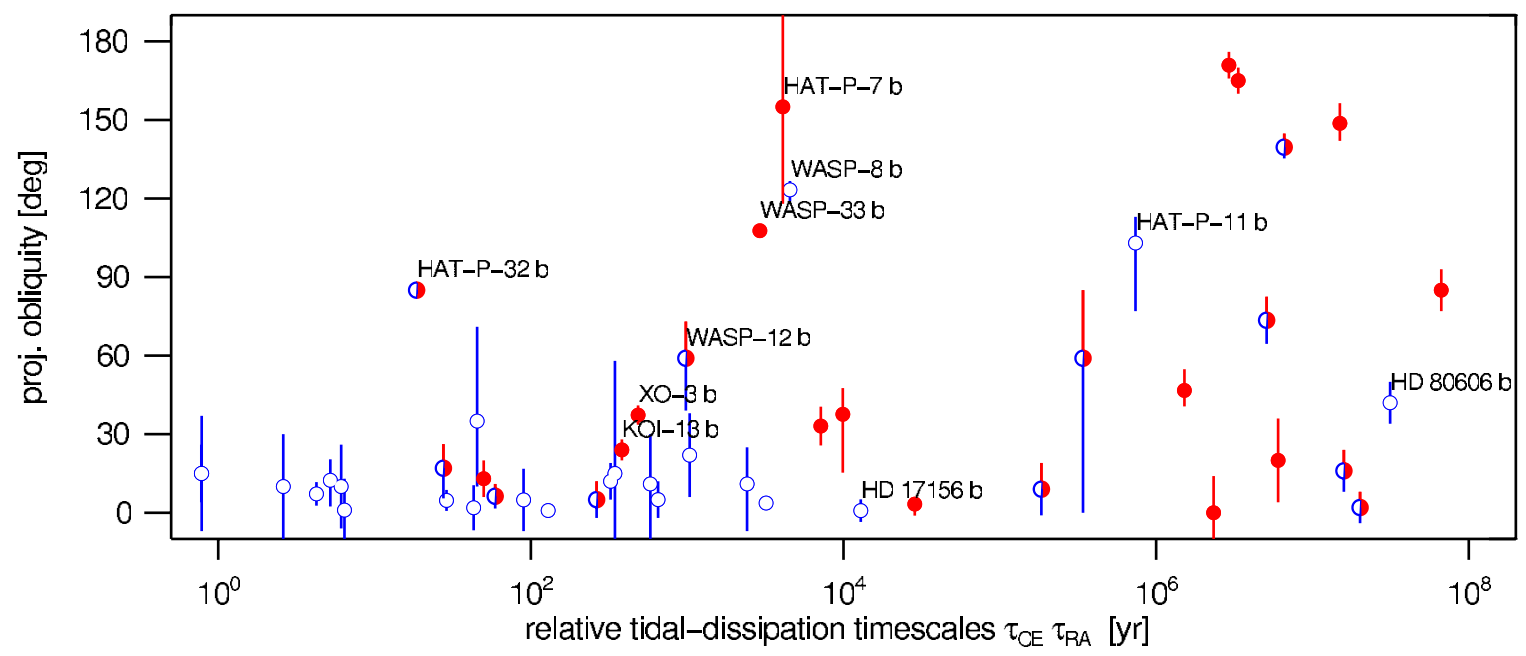

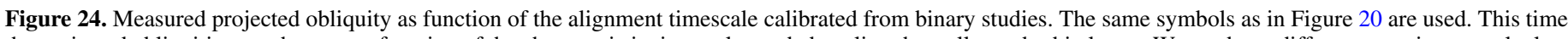

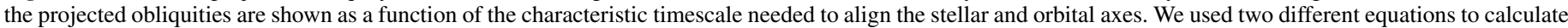

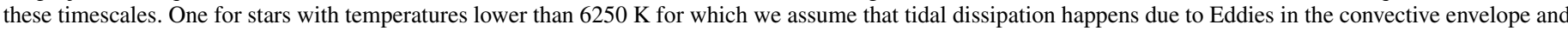

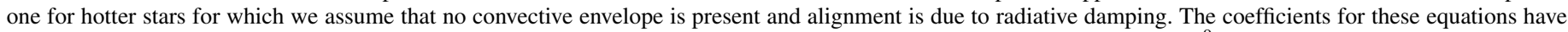
been calibrated with synchronization timescales in double star systems. Note that both timescales have been divided by $5 \times 10^{9}$.

(A color version of this figure is available in the online journal.)

direct the reader to Hansen 2012 for a different approach to this comparison). For the first approach we obtain the following relationships between the system parameters and the convective and radiative timescale for alignment from Zahn (1977, and references therein):

$$
\begin{gathered}
\frac{1}{\tau_{\mathrm{CE}}}=\frac{1}{10 \times 10^{9} \mathrm{yr}} q^{2}\left(\frac{a / R_{\star}}{40}\right)^{-6} \text { and }, \\
\frac{1}{\tau_{\mathrm{RA}}}=\frac{1}{0.25 \times 5 \times 10^{9} \mathrm{yr}} q^{2}(1+q)^{5 / 6}\left(\frac{a / R_{\star}}{6}\right)^{-17 / 2} .
\end{gathered}
$$

Here $q$ is the planet-to-star mass ratio. For stars with convective envelopes, synchronization is observed for binaries out to a scaled distance of $\sim 40$ during their main-sequence life, which we set to 10 Gyr for all "cool" stars. For "hot" stars, synchronization in binaries is observed out to $\sim 6$ times the scaled radius. Tidal damping is expected to be most efficient during the first quarter of their main-sequence life (Zahn 1977) (which we set here to 5 Gyr). See, e.g., Claret \& Cunha (1997) for a more recent comparison between theory and observations, mainly for stars of higher mass.

In Figure 24, we show the projected obliquity versus the characteristic timescale needed for realignment. For this we used Equation (2) for $T_{\text {eff }}<6250 \mathrm{~K}$ and Equation (3) for $T_{\text {eff }} \geqslant 6250 \mathrm{~K}$. (Both timescales were divided by $5 \times 10^{9}$ for normalization.) For most of the systems for which rapid alignment is expected, low projected obliquities are observed. For systems where tides are expected to be too slow in aligning and synchronizing stellar rotation, a very broad range of projected obliquities (apparently random) is observed.

We now return to the three apparent outliers from Figure 20: HAT-P-11, WASP-8, and HD 80606. While these are all stars with convective envelopes, the timescales for alignment are very long. This is because the scaled distances are greater than 15 (Figure 22). In the case of HAT-P-11, there is also the additional penalty from the relatively small planet mass $\left(q \approx 10^{-4}\right)$. Thus, in this light, these three "outliers" are not exceptions; they have high obliquities because the tidal timescales are very long.
There is one system which does seem to be an exception: HAT-P-32. The rotation axis of the star nearly lays in the plane of the orbit $\left(\lambda=85^{\circ} \pm 1.5\right)$, while all other systems with a similar tidal timescale do have projected obliquities consistent with good alignment. Because of the obliquity near $90^{\circ}$ tides couple only weakly to the stellar rotation (Lai 2012). The timescale for alignment in this system could be longer than estimated by our simple formula. If this is the only reason for the high obliquity in the HAT-P-32 system then we might expect to find more systems with short alignment timescales and similar obliquities in Figure 24, which do not exist in the current sample.

The measured effective temperature in HAT-P-32 is $6207 \pm$ $88 \mathrm{~K}$, and therefore we used Equation (2), applicable to stars with convective envelopes. If we would have assumed an effective temperature of $6250 \mathrm{~K}$, a value within the $1 \sigma$ interval of the measurement, and used Equation (3) then we would have obtained a $\tau_{\mathrm{RA}}$ of $1.5 \times 10^{5} \times 5 \times 10^{9} \mathrm{yr}$ instead of $\tau_{\mathrm{CE}}=1.8 \times 10^{1} \times 5 \times 10^{9} \mathrm{yr}$. This illustrates the aforementioned weakness of this first approach, that we have to make a binary decision on whether a star is "convective" or "radiative."

Note also that KOI-13 and XO-3 are "hot" stars which have significant misalignments, and yet they are found in between "cool" aligned systems. KOI-13 $\left(T_{\text {eff }}=8500 \mathrm{~K}\right)$ is hottest star in our sample and it is questionable if we can use the same tidal timescale for this system as for the other hot systems which are about $2000 \mathrm{~K}$ cooler (see Figure 20).

In the second approach we build upon Equation (2). Now we do not use any empirical calibration. For each planet-hosting star we estimate the mass contained in the convective envelope, and assume that the rate of energy dissipation is proportional to this convective mass,

$$
\frac{1}{\tau}=C \cdot \frac{1}{M_{\mathrm{cz}}} q^{2}\left(\frac{R}{a}\right)^{6},
$$

where $M_{\mathrm{cz}}$ indicates the mass contained in the outer convective zone and $C$ is an unspecified proportionality constant with units $\mathrm{g} \mathrm{s}^{-1}$. Our estimate for $M_{\mathrm{cz}}$ is based on the measured $T_{\text {eff }}$. This ensures a gradual decrease of tidal forces with increasing temperature. To establish the relation between $T_{\text {eff }}$ 


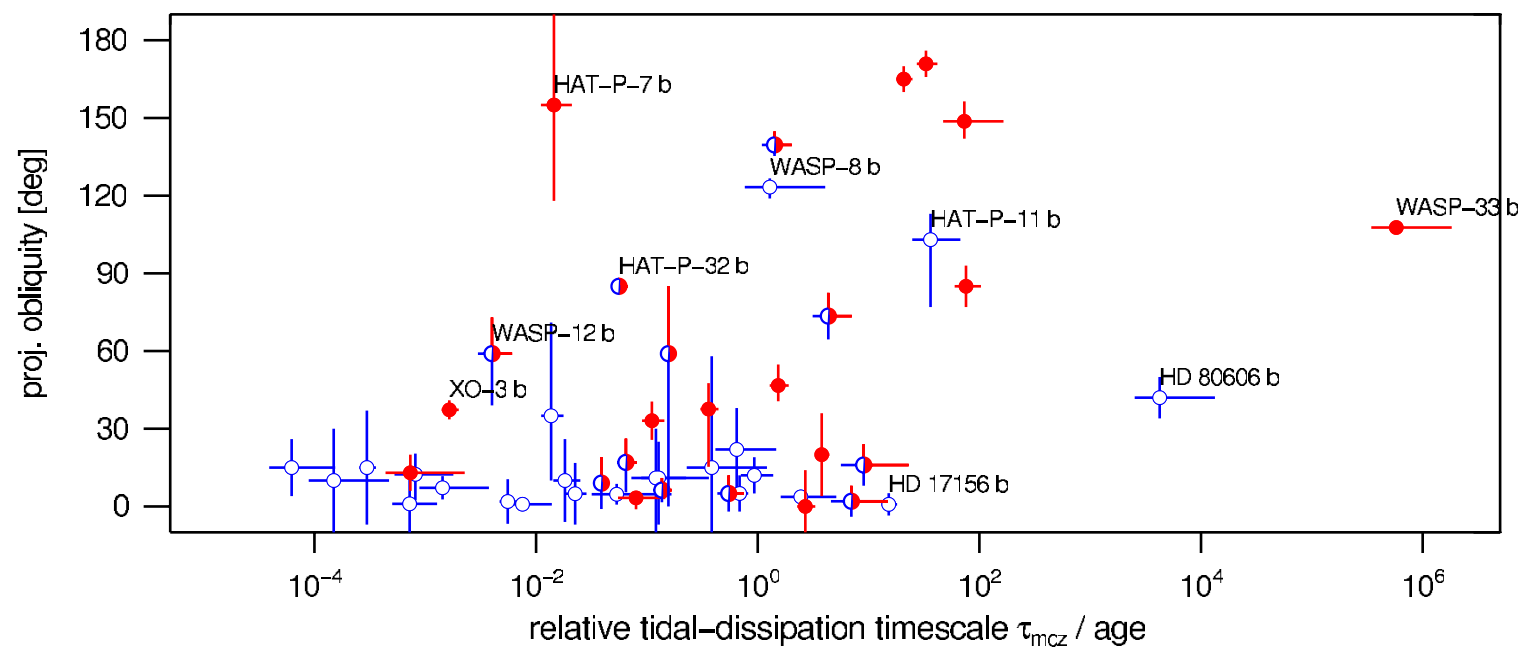

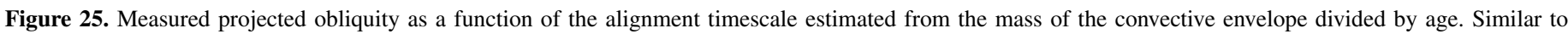

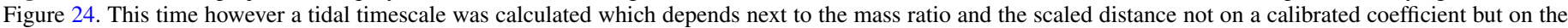

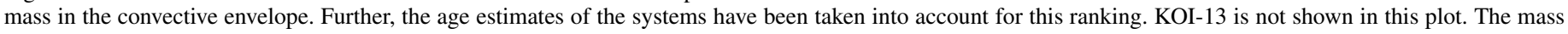
contained in its convective envelope is small and therefore according to Equation (4) it does practically not realign.

(A color version of this figure is available in the online journal.)

and convective mass we used the EZ-Web tool ${ }^{17}$ for stars with $T_{\text {eff }}<7000 \mathrm{~K}$, and the data from Pinsonneault et al. (2001) for hotter stars. To create Figure 25, we further divided the tidal timescale by the estimated main-sequence age, taking the uncertainty in the age estimate into account. As the ages are not well known, this leads to a substantial uncertainty in the positioning of a system on the horizontal axis. On average, the hot stars are younger than the cool stars. Therefore, the main effect of the division by age is a small shift of the hotter stars to the right side of the logarithmic plot.

The ordering of the cool stars is not substantially changed, relative to Figure 24, but there are now a few hot stars with significant obliquities and with similar tidal timescales as some cool aligned systems. The biggest outlier in this respect is HAT-P-7.

In summary, despite the shortcomings of our highly simplified approaches to calculating the theoretical tidal timescale, and a few exceptional cases, we do find support for the claim that the obliquities in hot Jupiter systems undergo damping by tidal dissipation. Systems with short tidal timescales are predominantly well-aligned, while systems with longer tidal timescales display an apparently random obliquity distribution. The implication is that the obliquities were once even more broadly distributed than we observe them today. Put differently, the "primordial" orbits of hot Jupiters (the orbits that existed shortly after the planets arrived close to the star) once had a very broad range of inclinations relative to the stellar equatorial plane.

\subsubsection{Angular Momentum Problem}

As Winn et al. (2010a) already pointed out, there is a theoretical problem with invoking tides in this context. The angular momentum in the stellar rotation compared to the angular momentum in the orbit (when the planet is close enough to significantly affect the stars rotation via tides) is so large that to synchronize and align the star the planet would surrender so much angular momentum that it would spiral into the star.

17 This tool is made available by Richard Townsend under the following url: http://www.astro.wisc.edu/ townsend
For nearly all systems in our sample the orbital velocity (at periastron) is larger than stellar rotation velocity. This causes trailing tides, and angular momentum is transported from the orbit toward the stellar rotation, leading to decay of the orbit (e.g., Levrard et al. 2009). Yet we see systems which have aligned axes and the planets have evidently survived.

To address this problem, Winn et al. (2010a) speculated that only the outer layers of the star synchronize and align with the orbit. In that case a smaller amount of the angular momentum would be transferred and the planetary inspiral would be avoided. It seems doubtful, though, that a separate rotation speed and rotation direction for the envelope relative to the stellar interior could be maintained for billions of years.

More recently, Lai (2012) suggested that the angular momentum problem is not as serious as it might seem. Given the complexities of tidal dynamics, he argued that there is no strong theoretical reason why the timescale for realignment must equal the timescale for synchronization, and indeed he provided a particular theoretical tidal model in which those timescales can differ by orders of magnitude. In his scenario the planets would first align the stellar rotation, and only much later speed up the rotation and spiral inward.

In this respect it is interesting that the tidal timescale calibrated via synchronization apparently sorts the systems consistently relative to each other, but the overall timescale is too long by orders of magnitude. As mentioned above we divided the timescales displayed in Figure 24 by $5 \times 10^{9}$. This could imply that realignment happens on a shorter timescale than synchronization. However, the calibration of the synchronization timescale was done with binary star systems having $q \approx 1$, and the tidal mechanism itself might be different for different regimes of its strength (Weinberg et al. 2012).

One might be able to test the hypothesis of Lai (2012) by seeking evidence for excess rotation in stars that are thought to have been realigned $\left(\lambda \approx 0^{\circ}\right)$. This could be done by measuring the stellar rotation period or $v \sin i_{\star}$ (if one is willing to assume $\sin i_{\star}$ is near unity in such systems). If an age estimate is also available, then one could employ the same approach as Schlaufman (2010) to assess whether or not the star is rotating at a typical rate, or if it is in the process of being spun up by the 
planet. This type of analysis was pursued by Pont (2009), though not with this specific hypothesis test in mind. This analysis could be profitably revisited now that many more systems are available for study. There is one caveat to this approach, which is that stars undergo very rapid spin evolution early in their lives due to disk locking and magnetic braking, i.e., for reasons unrelated to planets. If hot Jupiters arrive very early in the star's history, the realignment might happen in an epoch of rapid decrease of stellar rotation and any memory of an increased rotation due to tides raised by the planet might be lost.

The recent work by Hansen (2012) presented a calibration of the equilibrium tide theory using the measured parameters of hot Jupiter systems. While the approach taken in his work is different from ours, he arrived at similar conclusions to those described here: tides have shaped the obliquity distribution in these systems, and there is no theoretical need for core-envelope decoupling.

\subsection{High Obliquities: A Result of Dynamical Interactions, or Initially Inclined Disks?}

To interpret the finding that the host stars of hot Jupiters once had a broad distribution of obliquities, we must answer a crucial question. We need to know if the original obliquity is related to the existence of the hot Jupiter, or if stars and their protoplanetary disks are frequently misaligned for reasons unrelated to hot Jupiters. One might expect an initially close alignment between a star and its protoplanetary disk, as is observed in the Solar system and has been generally assumed in the exoplanet literature. However this is not a foregone conclusion, and indeed several authors have recently challenged this assumption, proposing that the Sun's low obliquity may be an atypical case.

Bate et al. (2010) proposed that a disk might become inclined with respect to the rotation axis of the central star, as a result of the complex accretion environment within a star cluster. In such a dense environment the tidal interaction with a companion star or other nearby stars could produce chaotic perturbations in the orbits of infalling material, with the material accreting later (destined to become planets) having a different orientation than the material that accreted earlier onto the star. Thies et al. (2011) studied the process of inclined infall of gas in detail and found that short period planets on eccentric and inclined orbits can be created in this way. A completely different mechanism for generating primordial misalignments was proposed by Lai et al. (2011), relying on a magnetic interaction between a young star and the inner edge of its accretion disk.

In these scenarios, the star has a high obliquity even though the planets may have never left the plane of the disk out of which they have formed, and therefore the measurements of obliquities bear information about the processes surrounding star formation rather than planet migration. How can one distinguish between misalignment created during the time the planet is still embedded in the disk or after the disk dissipated?

One approach, pursued by Watson et al. (2011), is to assess the degree of alignment between stars and their debris disks. Assuming that the stars as well as the debris disks trace the alignment of their predecessors one would learn about the degree of the alignment during the final stages of planet formation. Watson et al. (2011) found the inclinations of debris disks and their stars in a sample of nine systems to be consistent with good alignment. They caution that in their study only systems with $T_{\text {eff }}<6140 \mathrm{~K}$ have been observed and that misaligned system are found around hotter stars. However, as we have argued above, the found low obliquities that prevail around cool stars may be a consequence of tidal evolution and not of the mechanism which creates the obliquities.

Another approach is to measure the obliquities in binary star systems. If disks would be tilted relative to stellar rotation due to close encounters, then this could also lead to tilted rotation axes in double star systems. There should also be a trend toward misalignment with larger separation between the components in these systems. Conducting such measurements and seeking evidence for such trends is one of the goals of an ongoing observational program entitled BANANA (Binaries Are Not Always Neatly Aligned; Albrecht et al. 2011a).

A more direct way to answer the question raised in the preceding section has recently become possible, thanks to the discovery of systems with multiple transiting planets. A number of arguments support the idea that the orbital planes in such systems are closely aligned; most recently, Fabrycky et al. (2012) used the measured transit durations to show that the typical mutual inclinations are of order $2^{\circ}$. Therefore, it is reasonable to assume that the multiple planetary orbits trace the plane in which the planets formed. Any disruptive dynamical interactions, such as those which have been proposed to explain hot Jupiters, would likely have produced higher mutual inclinations in the multiple-transiting systems. Under that assumption, RM measurements (or other measures of obliquity) in those multiple-transiting systems would establish the angle between the circumstellar disk and the stellar equator. If good alignment is found to be the rule, then the high obliquities in hot Jupiter systems would be more readily interpreted as a consequence of planet migration than as primordial star-disk misalignments.

\section{SUMMARY}

In this paper, we have presented new observations of the RM effect for 14 systems harboring hot Jupiters. In addition we critically reviewed the literature, in some cases re-analyzing data that had been obtained previously in order to conform with our protocols. We then used these data to show that the distribution in obliquities is consistent with being shaped by tides raised by the hot Jupiters on the stars. For this we revisited the correlation between the projected obliquity and the effective temperature discovered by Winn et al. (2010a), now with a sample of RM measurements twice as large as was then available. We showed that the new measurements agree with the pattern proposed by Winn et al. (2010a). With the enlarged sample we showed that obliquity in systems with close-in massive planets further depend on the mass ratio and the distance between star and planet, in roughly the manner expected if tides are responsible for the low obliquities.

Motivated by these results we then devised two different parameters that represent, at least crudely, the theoretical tidal timescales. This showed that systems which are expected to align fast are all showing projections of the obliquities which are consistent with good alignment. In contrast, systems for which tidal interaction is expected to be weak, due to the stellar structure, distance, or mass ratio, show a nearly random distribution of projected obliquities. Our interpretation is that stars with hot Jupiters once had a very broad range of obliquities. It is tempting to argue further that the large obliquities originate from the same process that produces hot Jupiters, thereby favoring explanations involving dynamical scattering or the Kozai effect, and disfavoring the gradual inspiral due to torques in a protoplanetary disk. However, more observations are needed 
to check on the possibility that stars and their disks are frequently misaligned for reasons unrelated to hot Jupiters. Among these observations are the extension of RM studies to planets other than hot Jupiters, and measurements of obliquities in binary star systems and in systems with multiple transiting planets.

The authors are grateful to Nevin Weinberg, Dan Fabrycky, Smadar Naoz, Amaury Triaud, and René Heller for comments on the manuscript. Work by S.A. and J.N.W. was supported by NASA Origins award NNX09AB33G and NSF grant No. 1108595. G.B. and J.H. acknowledge the support from grants NSF AST-1108686 and NASA NNX09AB29G. T.H. is supported by Japan Society for Promotion of Science (JSPS) Fellowship for Research (DC1: 22-5935). This research has made use of the following web resources: simbad.u-strasbg.fr, adswww.harvard.edu,arxiv.org. The W. M. Keck Observatory is operated as a scientific partnership among the California Institute of Technology, the University of California, and the National Aeronautics and Space Administration, and was made possible by the generous financial support of the W. M. Keck Foundation. We extend special thanks to those of Hawaiian ancestry on whose sacred mountain of Mauna Kea we are privileged to be guests.

\section{REFERENCES}

Albrecht, S., Reffert, S., Snellen, I., Quirrenbach, A., \& Mitchell, D. S. 2007, A\&A, 474, 565

Albrecht, S., Winn, J. N., Butler, R. P., et al. 2012, ApJ, 744, 189

Albrecht, S., Winn, J. N., Carter, J. A., Snellen, I. A. G., \& de Mooij, E. J. W. 2011a, ApJ, 726, 68

Albrecht, S., Winn, J. N., Johnson, J. A., et al. 2011b, ApJ, 738, 50

Anderson, D. R., Collier Cameron, A., Gillon, M., et al. 2011a, A\&A, 534, A16 Anderson, D. R., Collier Cameron, A., Hellier, C., et al. 2011b, A\&A, 531, A60 Anderson, D. R., Smith, A. M. S., Lanotte, A. A., et al. 2011c, MNRAS, 416, 2108

Bakos, G. Á., Hartman, J. D., Torres, G., et al. 2012, AJ, 144, 19

Barnes, J. W., Linscott, E., \& Shporer, A. 2011, ApJS, 197, 10

Barnes, S. A. 2003, ApJ, 586, 464

Bate, M. R., Lodato, G., \& Pringle, J. E. 2010, MNRAS, 401, 1505

Bouchy, F., Queloz, D., Deleuil, M., et al. 2008, A\&A, 482, L25

Brown, D. J. A., Cameron, A. C., Anderson, D. R., et al. 2012, MNRAS, 423, 1503

Brown, T. M., Charbonneau, D., Gilliland, R. L., Noyes, R. W., \& Burrows, A. 2001, ApJ, 552, 699

Buchhave, L. A., Bakos, G. Á., Hartman, J. D., et al. 2010, ApJ, 720, 1118

Butler, R. P., Marcy, G. W., Williams, E., et al. 1996, PASP, 108, 500

Butler, R. P., Vogt, S. S., Marcy, G. W., et al. 2004, ApJ, 617, 580

Carter, J. A., Winn, J. N., Gilliland, R., \& Holman, M. J. 2009, ApJ, 696, 241

Carter, J. A., Yee, J. C., Eastman, J., Gaudi, B. S., \& Winn, J. N. 2008, ApJ, 689,499

Claret, A. 2000, A\&A, 363, 1081

Claret, A., \& Cunha, N. C. S. 1997, A\&A, 318, 187

Collier Cameron, A., Bruce, V. A., Miller, G. R. M., Triaud, A. H. M. J., \& Queloz, D. 2010, MNRAS, 403, 151

Crane, J. D., Shectman, S. A., Butler, R. P., et al. 2010, Proc. SPIE, 7735, 170

Cresswell, P., Dirksen, G., Kley, W., \& Nelson, R. P. 2007, A\&A, 473, 329

Deleuil, M., Deeg, H. J., Alonso, R., et al. 2008, A\&A, 491, 889

Désert, J.-M., Charbonneau, D., Demory, B.-O., et al. 2011, ApJS, 197, 14

Dragomir, D., Kane, S. R., Pilyavsky, G., et al. 2011, AJ, 142, 115

Fabrycky, D., \& Tremaine, S. 2007, ApJ, 669, 1298

Fabrycky, D. C., Lissauer, J. J., Ragozzine, D., et al. 2012, arXiv:1202.6328

Fabrycky, D. C., \& Winn, J. N. 2009, ApJ, 696, 1230

Gandolfi, D., Hébrard, G., Alonso, R., et al. 2010, A\&A, 524, A55

Gaudi, B. S., \& Winn, J. N. 2007, ApJ, 655, 550

Gillon, M., Anderson, D. R., Triaud, A. H. M. J., et al. 2009, A\&A, 501, 785

Gillon, M., Lanotte, A. A., Barman, T., et al. 2010, A\&A, 511, A3

Gillon, M., Pont, F., Demory, B.-O., et al. 2007, A\&A, 472, L13

Gray, D. F. 1984, ApJ, 281, 719

Guenther, E. W., Díaz, R. F., Gazzano, J.-C., et al. 2012, A\&A, 537, A136

Hansen, B. 2012, arXiv:1204.3903
Hartman, J. D., Bakos, G. Á., Torres, G., et al. 2011, ApJ, 742, 59

Hebb, L., Collier-Cameron, A., Loeillet, B., et al. 2009, ApJ, 693, 1920

Hebb, L., Collier-Cameron, A., Triaud, A. H. M. J., et al. 2010, ApJ, 708, 224

Hébrard, G., Bouchy, F., Pont, F., et al. 2008, A\&A, 488, 763

Hébrard, G., Désert, J.-M., Díaz, R. F., et al. 2010, A\&A, 516, A95

Hébrard, G., Ehrenreich, D., Bouchy, F., et al. 2011a, A\&A, 527, L11

Hébrard, G., Evans, T. M., Alonso, R., et al. 2011b, A\&A, 533, A130

Hellier, C., Anderson, D. R., Collier-Cameron, A., et al. 2011, ApJ, 730, L31

Hellier, C., Anderson, D. R., Gillon, M., et al. 2009, ApJ, 690, L89

Hirano, T., Narita, N., Sato, B., et al. 2011a, PASJ, 63, L57

Hirano, T., Narita, N., Shporer, A., et al. 2011b, PASJ, 63, 531

Hirano, T., Suto, Y., Taruya, A., et al. 2010, ApJ, 709, 458

Hirano, T., Suto, Y., Winn, J. N., et al. 2011c, ApJ, 742, 69

Hosokawa, Y. 1953, PASJ, 5, 88

Husnoo, N., Pont, F., Hébrard, G., et al. 2011, MNRAS, 413, 2500

Jenkins, J. M., Borucki, W. J., Koch, D. G., et al. 2010, ApJ, 724, 1108

Johnson, J. A., Winn, J. N., Albrecht, S., et al. 2009, PASP, 121, 1104

Johnson, J. A., Winn, J. N., Bakos, G. Á., et al. 2011, ApJ, 735, 24

Johnson, J. A., Winn, J. N., Narita, N., et al. 2008, ApJ, 686, 649

Kipping, D. M., Bakos, G. Á., Hartman, J., et al. 2010, ApJ, 725, 2017

Lai, D. 2012, MNRAS, 423, 486

Lai, D., Foucart, F., \& Lin, D. N. C. 2011, MNRAS, 412, 2790

Laughlin, G., Marcy, G. W., Vogt, S. S., Fischer, D. A., \& Butler, R. P. 2005, ApJ, 629, L121

Levrard, B., Winisdoerffer, C., \& Chabrier, G. 2009, ApJ, 692, L9

Lin, D. N. C., Bodenheimer, P., \& Richardson, D. C. 1996, Nature, 380, 606

Lister, T. A., Anderson, D. R., Gillon, M., et al. 2009, ApJ, 703, 752

Loeillet, B., Shporer, A., Bouchy, F., et al. 2008, A\&A, 481, 529

Maciejewski, G., Errmann, R., Raetz, S., et al. 2011, A\&A, 528, A65

Mandel, K., \& Agol, E. 2002, ApJ, 580, L171

Maness, H. L., Marcy, G. W., Ford, E. B., et al. 2007, PASP, 119, 90

Mazeh, T. 2008, in EAS Publications Series, Vol. 29, ed. M.-J. Goupil

\& J.-P. Zahn (Cambridge: Cambridge Univ. Press), 1, http://www.easjournal.org/action/displayAbstract?fromPage $=$ online \&aid $=8461965$

McLaughlin, D. B. 1924, ApJ, 60, 22

Mislis, D., \& Hodgkin, S. 2012, MNRAS, 422, 1512

Morton, T. D., \& Johnson, J. A. 2011, ApJ, 729, 138

Moutou, C., Díaz, R. F., Udry, S., et al. 2011, A\&A, 533, A113

Nagasawa, M., Ida, S., \& Bessho, T. 2008, ApJ, 678, 498

Naoz, S., Farr, W. M., Lithwick, Y., Rasio, F. A., \& Teyssandier, J. 2011, Nature, 473, 187

Narita, N., Enya, K., Sato, B., et al. 2007, PASJ, 59, 763

Narita, N., Hirano, T., Sato, B., et al. 2009a, PASJ, 61, 991

Narita, N., Hirano, T., Sato, B., et al. 2011, PASJ, 63, L67

Narita, N., Sato, B., Hirano, T., \& Tamura, M. 2009b, PASJ, 61, L35

Narita, N., Sato, B., Hirano, T., et al. 2010, PASJ, 62, 653

Noyes, R. W., Bakos, G. Á., Torres, G., et al. 2008, ApJ, 673, L79

Ohta, Y., Taruya, A., \& Suto, Y. 2005, ApJ, 622, 1118

Pál, A., Bakos, G. Á., Torres, G., et al. 2008, ApJ, 680, 1450

Pál, A., Bakos, G. Á., Torres, G., et al. 2010, MNRAS, 401, 2665

Pinsonneault, M. H., DePoy, D. L., \& Coffee, M. 2001, ApJ, 556, L59

Pont, F. 2009, MNRAS, 396, 1789

Pont, F., Endl, M., Cochran, W. D., et al. 2010, MNRAS, 402, L1

Queloz, D., Anderson, D., Collier Cameron, A., et al. 2010, A\&A, 517, L1

Queloz, D., Eggenberger, A., Mayor, M., et al. 2000, A\&A, 359, L13

Rossiter, R. A. 1924, ApJ, 60, 15

Sanchis-Ojeda, R., Winn, J. N., Holman, M. J., et al. 2011, ApJ, 733, 127

Sato, B., Fischer, D. A., Henry, G. W., et al. 2005, ApJ, 633, 465

Schlaufman, K. C. 2010, ApJ, 719, 602

Shporer, A., \& Brown, T. 2011, ApJ, 733, 30

Shporer, A., Jenkins, J. M., Rowe, J. F., et al. 2011, AJ, 142, 195

Simpson, E. K., Pollacco, D., Cameron, A. C., et al. 2011, MNRAS, 414, 3023

Smalley, B., Anderson, D. R., Collier Cameron, A., et al. 2010, A\&A, 520, A56

Southworth, J., Hinse, T. C., Dominik, M., et al. 2009, ApJ, 707, 167

Southworth, J., Hinse, T. C., Dominik, M., et al. 2010, ApJ, 723, 1829

Szabó, G. M., Szabó, R., Benkő, J. M., et al. 2011, ApJ, 736, L4

Szentgyorgyi, A. H., Geary, J. G., Latham, D. W., et al. 2005, BAAS, 37, 1339

Thies, I., Kroupa, P., Goodwin, S. P., Stamatellos, D., \& Whitworth, A. P. 2011, MNRAS, 417, 1817

Torres, G., Andersen, J., \& Giménez, A. 2010, A\&AR, 18, 67

Torres, G., Winn, J. N., \& Holman, M. J. 2008, ApJ, 677, 1324

Triaud, A. H. M. J. 2011, A\&A, 534, L6

Triaud, A. H. M. J., Collier Cameron, A., Queloz, D., et al. 2010, A\&A, 524, A25

Triaud, A. H. M. J., Queloz, D., Bouchy, F., et al. 2009, A\&A, 506, 377

Triaud, A. H. M. J., Queloz, D., Hellier, C., et al. 2011, A\&A, 531, A24

Tripathi, A., Winn, J. N., Johnson, J. A., et al. 2010, ApJ, 715, 421 
Valenti, J. A., \& Fischer, D. A. 2005, ApJS, 159, 141

Vogt, S. S., Allen, S. L., Bigelow, B. C., et al. 1994, Proc. SPIE, 2198, 362

Watson, C. A., Littlefair, S. P., Diamond, C., et al. 2011, MNRAS, 413, L71

Weinberg, N. N., Arras, P., Quataert, E., \& Burkart, J. 2012, ApJ, 751, 136

Winn, J. N., Albrecht, S., Johnson, J. A., et al. 2011a, ApJ, 741, L1

Winn, J. N., Fabrycky, D., Albrecht, S., \& Johnson, J. A. 2010a, ApJ, 718, L145

Winn, J. N., Howard, A. W., Johnson, J. A., et al. 2011b, AJ, 141, 63

Winn, J. N., Johnson, J. A., Albrecht, S., et al. 2009, ApJ, 703, L99

Winn, J. N., Johnson, J. A., Howard, A. W., et al. 2010b, ApJ, 718, 575
Winn, J. N., Johnson, J. A., Howard, A. W., et al. 2010c, ApJ, 723, L223

Winn, J. N., Johnson, J. A., Narita, N., et al. 2008, ApJ, 682, 1283

Winn, J. N., Johnson, J. A., Peek, K. M. G., et al. 2007, ApJ, 665, L167

Winn, J. N., Noyes, R. W., Holman, M. J., et al. 2005, ApJ, 631, 1215

Wolf, A. S., Laughlin, G., Henry, G. W., et al. 2007, ApJ, 667, 549

Zahn, J.-P. 1977, A\&A, 57, 383

Zahn, J.-P. 2008, in EAS Publications Series, Vol. 29, ed. M.-J. Goupil \& J.-P. Zahn (Cambridge: Cambridge Univ. Press), 67, http://www.easjournal.org/action/displayAbstract?fromPage $=$ online $\&$ aid $=8461968$ 